Supporting information

\title{
Iridium(VII)-Corrole Terminal Carbides Should Exist as Stable Compounds
}

\author{
Jeanet Conradie, ${ }^{\mathrm{a}, \mathrm{b}}$ Abraham B. Alemayehu ${ }^{\mathrm{a}}$ and Abhik Ghosh*,a \\ ${ }^{a}$ Department of Chemistry, UiT - The Arctic University of Norway, N-9037 Tromsø, \\ Norway; Email: abhik.ghosh@uit.no \\ ${ }^{\mathrm{b}}$ Department of Chemistry, University of the Free State, P.O. Box 339, Bloemfontein 9300, \\ Republic of South Africa.
}




\section{Table of Contents}

A. Computational method $\ldots \ldots \ldots \ldots \ldots \ldots \ldots \ldots \ldots \ldots \ldots \ldots$

B. Example input file $\ldots \ldots \ldots \ldots \ldots \ldots \ldots \ldots \ldots \ldots \ldots \ldots \ldots$

C. Optimized all-electron OLYP/ZORA-STO-TZ2P Cartesian coordinates

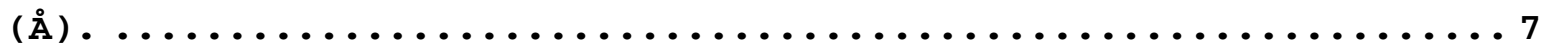

1. $\operatorname{Ir}\left[\right.$ Cor] $(\mathrm{C}) ; C_{\mathrm{s}} ; S=0$; Total bonding energy $=$

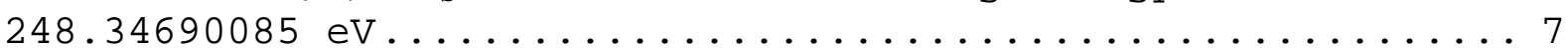

2. $\operatorname{Ir}[$ Cor $](C) ; C_{s} ; S=1 ;$ Total bonding energy $=-$

246.54046220 ev . . . . . . . . . . . . . . . . . . . . . . . . . . 7

3. $\{\operatorname{Ir}[\text { Cor }](\mathrm{C})\}^{-} ; C_{\mathrm{s}} ; S=1 / 2 ;$ Total bonding energy $=$

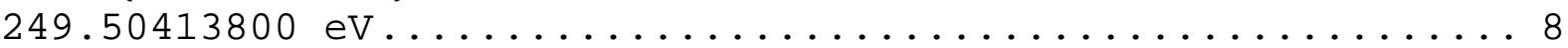

4. $\operatorname{Ir}[\mathrm{Cor}](\mathrm{NMe})_{2} ; C_{2 v} ; S=0 ;$ Total bonding energy $=$

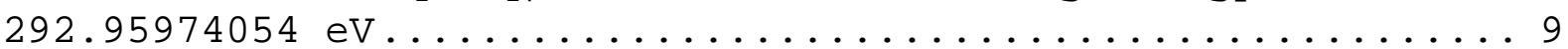

5. $\operatorname{Ir}[\mathrm{Cor}](\mathrm{NMe})_{2} ; C_{2 v} ; S=1 ;$ Total bonding energy $=$

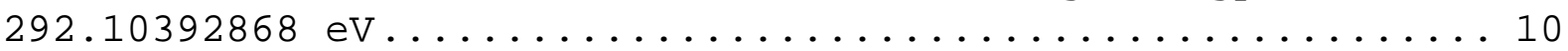

6. $\left\{\operatorname{Ir}[\mathrm{Cor}](\mathrm{NMe})_{2}\right\} ; C_{2 v} ; S=1 / 2 ;$ Total bonding energy $=-$ -

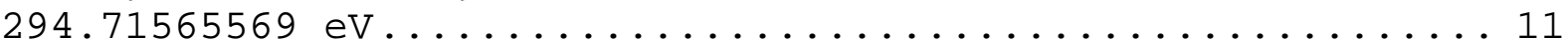

7. $\operatorname{Re}[$ Cor $](C) ; C_{s} ; S=0$; Total bonding energy $=-$

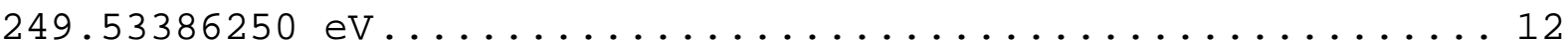

8. $\operatorname{Re}[$ Cor $](C) ; C_{s} ; S=1$; Total bonding energy $=-$

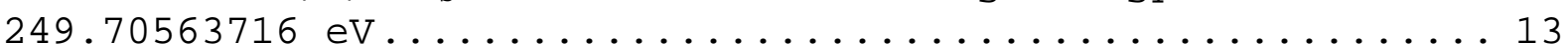

9. $\{\operatorname{Re}[\operatorname{Cor}](\mathrm{C})\}^{-} ; C_{\mathrm{s}} ; S=1 / 2 ;$ Total bonding energy $=$

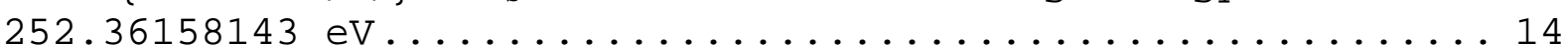

10. $\operatorname{Re}[\mathrm{Cor}](\mathrm{NMe})_{2} ; C_{2 v} ; S=0$; Total bonding energy $=$

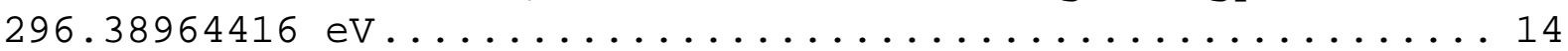

11. $\operatorname{Re}[\mathrm{Cor}](\mathrm{NMe})_{2} ; C_{2 v} ; S=1 ;$ Total bonding energy $=$

$295.84740414 \mathrm{eV}$ (non Aufbau solution) ................ 15

12. $\left\{\operatorname{Re}[\mathrm{Cor}](\mathrm{NMe})_{2}\right\}^{-} ; C_{2 v} ; S=1 / 2 ;$ Total bonding energy $=-$

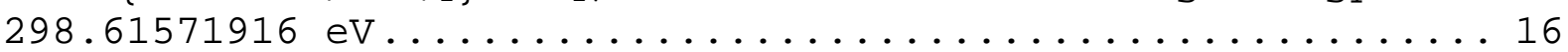

13. $\operatorname{Ir}[\mathrm{Cor}]\left(\mathrm{PMe}_{3}\right) ; C_{\mathrm{s}} ; S=0 ;$ Total bonding energy $=$

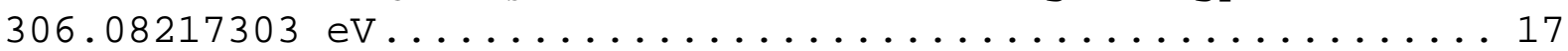

14. $\operatorname{Ir}[\mathrm{Cor}](\mathrm{CO}) ; C_{\mathrm{s}} ; S=0 ;$ Total bonding energy $=-$

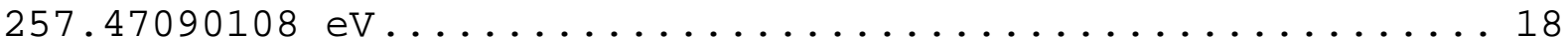

15. $\operatorname{Ir}[\mathrm{Cor}](\mathrm{CO})\left(\mathrm{PMe}_{3}\right) ; C_{\mathrm{s}} ; S=0$; Total bonding energy $=-$

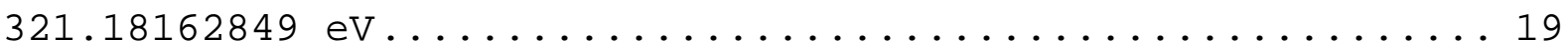

16. $\operatorname{Re}[$ Cor] (NMe); $C$ s; $S=0$; Total bonding energy $=-$

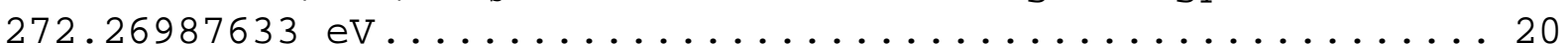

17. $\mathrm{Me}_{3} \mathrm{PO} ; C_{\mathrm{s}} ; S=0$; Total bonding energy $=-71.13474396$ eV 21

18. $C 0 ; C_{\text {lin }} ; S=0$; Total bonding energy $=-14.68008335 \mathrm{eV} .21$

19. $\mathrm{PMe}_{3} ; C_{\mathrm{s}} ; S=0$; Total bonding energy $=-63.38945444 \mathrm{eV} .22$

20. $\mathrm{MeN}=\mathrm{NMe} ; C_{2 \mathrm{~h}} ; S=0$; Total bonding energy $=-53.36675122 \mathrm{eV}$ 22

21. $\mathrm{Me}_{3} \mathrm{P}=\mathrm{NMe} ; C_{1} ; S=0$; Total bonding energy $=-90.40548752 \mathrm{eV}$ 22

D. Optimized all-electron B3LYP/ZORA-STO-TZ2P Cartesian coordinates

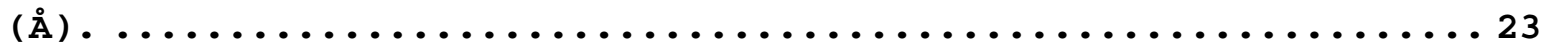

22. $\operatorname{Ir}[$ Cor $](C) ; C$ s $S=0$; Total bonding energy $=-$

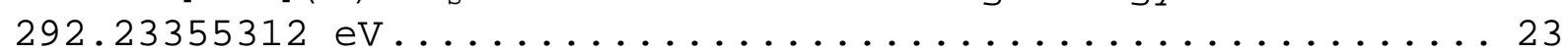

23. $\operatorname{Ir}[$ Cor $](\mathrm{C}) ; C_{\mathrm{s}} ; S=1$; Total bonding energy $=-$

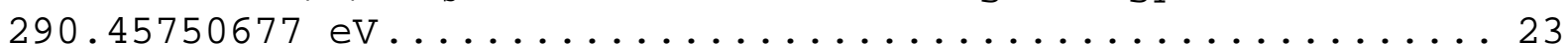


24. $\{\operatorname{Ir}[\operatorname{Cor}](\mathrm{C})\}^{-} ; C_{\mathrm{s}} ; S=1 / 2 ;$ Total bonding energy $=-$ -

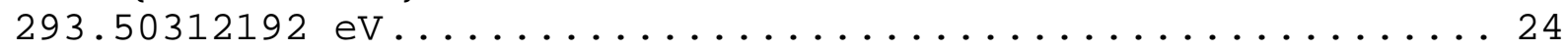

25. $\operatorname{Ir}[\mathrm{Cor}](\mathrm{NMe})_{2} ; C_{2 v} ; S=0 ;$ Total bonding energy $=-\cdots$

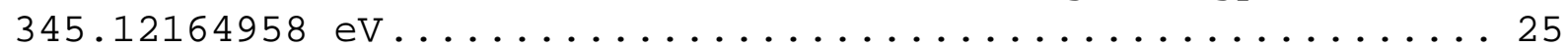
26. $\operatorname{Ir}[\mathrm{Cor}](\mathrm{NMe})_{2} ; C_{2 v} ; S=1 ;$ Total bonding energy $=-\cdots$

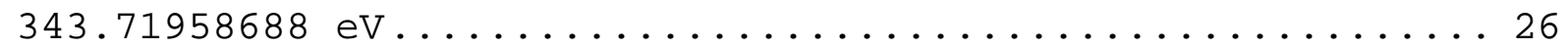
27. $\left\{\operatorname{Ir}[\mathrm{Cor}](\mathrm{NMe})_{2}\right\} ; C_{2 v} ; S=1 / 2 ;$ Total bonding energy $=-\cdots$

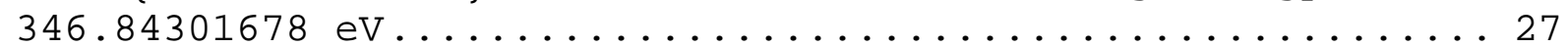
28. $\operatorname{Re}[\mathrm{Cor}](\mathrm{C}) ; C_{\mathrm{s}} ; S=0 ;$ Total bonding energy $=-$

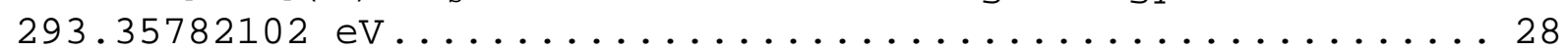
29. $\operatorname{Re}[$ Cor $](C) ; C_{s} ; S=1$; Total bonding energy $=-$

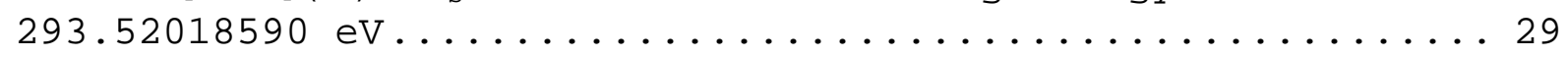
30. $\{\operatorname{Re}[\operatorname{Cor}](\mathrm{C})\}^{-} ; C_{s} ; S=1 / 2 ;$ Total bonding energy $=-$

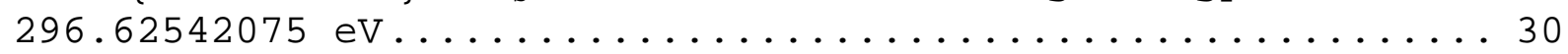
31. $\operatorname{Re}[\mathrm{Cor}](\mathrm{NMe})_{2} ; C_{2 v} ; S=0 ;$ Total bonding energy $=-$

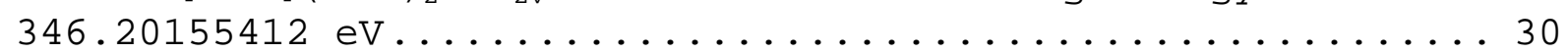
32. $\operatorname{Re}[\mathrm{Cor}](\mathrm{NMe})_{2} ; C_{2 v} ; S=1 ;$ Total bonding energy $=-\cdots$

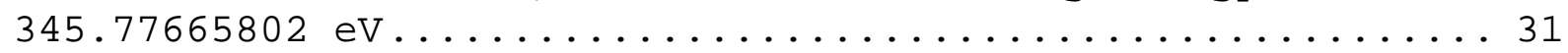
33. $\left\{\operatorname{Re}[\mathrm{Cor}](\mathrm{NMe})_{2}\right\}^{-} ; C_{2 v} ; S=1 / 2 ;$ Total bonding energy $=-$

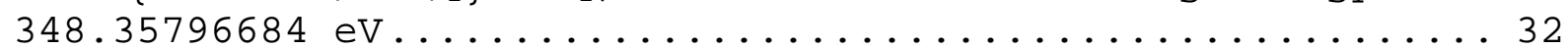
34. $\operatorname{Ir}[\mathrm{Cor}]\left(\mathrm{PMe}_{3}\right) ; C_{s} ; S=0 ;$ Total bonding energy $=-$

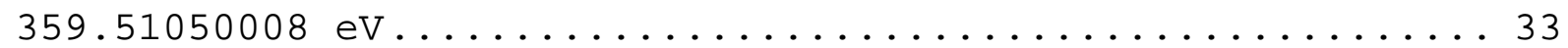
35. $\operatorname{Ir}[\mathrm{Cor}](\mathrm{CO}) ; C_{s} ; S=0 ;$ Total bonding energy $=-\cdot$

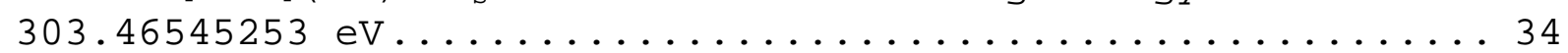
36. $\operatorname{Ir}[\mathrm{Cor}](\mathrm{CO})\left(\mathrm{PMe}_{3}\right) ; C_{s} ; S=0 ;$ Total bonding energy $=$ - -

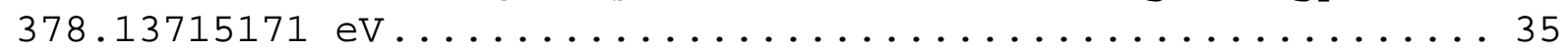
37. $\operatorname{Re}[\mathrm{Cor}](\mathrm{NMe}) ; C_{s} ; S=0 ;$ Total bonding energy $=-$

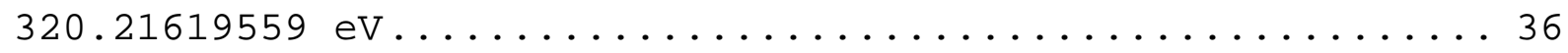
38. $\mathrm{Me}_{3} \mathrm{PO} ; C_{s} ; S=0$; Total bonding energy $=-83.70709718$ eV 37 39. $\mathrm{Co} ; C_{\text {lin }} ; S=0$; Total bonding energy $=-18.10684518 \mathrm{eV} .38$ 40. $\mathrm{PMe}_{3} ; C_{s} ; S=0$; Total bonding energy $=-73.88983796 \mathrm{eV} .38$ 41. MeN=NMe; $C_{2 h} ; S=0 ;$ Total bonding energy $=-63.42932681 \mathrm{eV}$ 38

42. $\mathrm{Me}_{3} \mathrm{P}=\mathrm{NMe} ; C_{1} ; S=0 ;$ Total bonding energy $=-105.95446898$ eV 38 


\section{A. Computational method}

All DFT calculations were carried out with the ADF 2018 program release r74086 2019-04-17,1 the ZORA Hamiltonian, fine integration grids, and tight criteria for SCF cycles and geometry optimizations. All optimizations were done in the gas phase with scalarrelativistic DFT calculations with the OLYP2,3 and B3LYP 4,5 functionals and all-electron ZORA6,7-STO-TZ2P basis sets. Additional details are provided in the sample input ( $p$ S5). Note that ADF does not provide conventional total energies, but rather total bonding energies, which are energies relative to those of the spherical, spin-restricted atoms. For calculating energy differences between two species, total bonding energies can be used exactly like total energies. See the ADF manual for further details.

( $\left.{ }^{1}\right)$ Velde, G. T.; Bickelhaupt, F. M.; Baerends, E. J.; Guerra, C. F.; van Gisbergen, S. J. A.; Snijders, J. G.; Ziegler, T. Chemistry with ADF. J. Comput. Chem. 2001, 22, 931-967.

$\left({ }^{2}\right)$ Handy, N. C.; Cohen, A. Left-Right Correlation Energy. J. Mol. Phys. 2001, 99, 403-412.

$\left({ }^{3}\right)$ T Lee, C.T.; Yang, W.T.; Parr, R.G. Development of the ColleSalvetti Correlation-Energy Formula into a Functional of the Electron-Density. Phys. Rev. B, 1988, 37, 785-789.

$\left({ }^{4}\right)$ Becke, A. D. A New Mixing of Hartree-Fock and Local

Density-Functional Theories. J. Chem. Phys. 1993, 98, 1372-1377.

$\left({ }^{5}\right)$ Stephens, P. J.; Devlin, F. J.; Chabalowski, C. F.; Frisch, M. J. $A b$ Initio Calculation of Vibrational Absorption and Circular Dichroism Spectra Using Density Functional Force Fields. J. Phys. Chem. 1994, 98, 11623-11627.

$\left({ }^{6}\right)$ van Lenthe, E.; Baerends, E. J.; Snijders, J. G. Relativistic Regular Two-Component Hamiltonians. J. Chem. Phys. 1993, 99, 45974610 .

$\left({ }^{7}\right)$ van Lenthe, E.; Baerends, E. J.; Snijders, J. G. Relativistic Total Energy Using Regular Approximations. J. Chem. Phys. 1994, 101, 9783-9792. 


\title{
B. Example input file
}

Title $\operatorname{Ir}[\mathrm{Cor}](\mathrm{NMe}) 2, \mathrm{C} 2 \mathrm{v}, \mathrm{S}=0$

\section{COMMENT}

END

\author{
UNITS \\ length angstrom \\ END
}

\begin{tabular}{|c|c|}
\hline oms & Cartesian \\
\hline $\mathrm{N}$ & $-\odot .01707000 \odot$ \\
\hline $\mathrm{N}$ & $\odot .834731000$ \\
\hline $\mathrm{N}$ & $\odot .834731000$ \\
\hline $\mathrm{N}$ & $-\odot .017070 \odot \odot \odot$ \\
\hline C & $-\odot .38879300 \odot$ \\
\hline C & $-\odot .7213290 \odot \odot$ \\
\hline C & $\odot .06467400 \odot$ \\
\hline C & $-\odot .486793000$ \\
\hline C & $\odot .48220300 \odot$ \\
\hline C & $-\odot .6980990 \odot \odot$ \\
\hline C & $\odot .844259000$ \\
\hline C & $-\odot .48679300 \odot$ \\
\hline C & 1.211783000 \\
\hline C & $-\odot .72132900 \odot$ \\
\hline C & $1.42780400 \odot$ \\
\hline C & 1.211783000 \\
\hline C & 1.184811000 \\
\hline C & $\odot .84425900 \odot$ \\
\hline C & $\odot .48220300 \odot$ \\
\hline C & 1.184811000 \\
\hline C & $\odot .064674000$ \\
\hline C & 1.427804000 \\
\hline C & $-\odot .38879300 \odot$ \\
\hline C & $2.5787540 \odot \odot$ \\
\hline Ir & $\odot .63624800 \odot$ \\
\hline $\mathrm{H}$ & 1.704283000 \\
\hline $\mathrm{H}$ & 1.277023000 \\
\hline $\mathrm{H}$ & $1.7042830 \odot \odot$ \\
\hline $\mathrm{H}$ & 1.277023000 \\
\hline $\mathrm{H}$ & $\odot .494102000$ \\
\hline $\mathrm{H}$ & $-\odot .45881400 \odot$ \\
\hline $\mathrm{H}$ & -1.105910000 \\
\hline $\mathrm{H}$ & -1.074925000 \\
\hline $\mathrm{H}$ & -1.105910000 \\
\hline $\mathrm{H}$ & $-\odot .45881400 \odot$ \\
\hline $\mathrm{H}$ & $\odot .4941020 \odot \odot$ \\
\hline
\end{tabular}

$-0.939209000$

1.598057000

1.598057000

$-0.939209000$

$-1.854338000$

$-2.803735000$

$-0.685444000$

$-2.225779000$

0.525278000

$-2.830289000$

1.644781000

$-2.225779000$

2.982936000

$-2.803735000$

3.706942000

2.982936000

2. 818760000

1.644781000

$\odot .525278000$

2.818760000

$-0.685444000$

3.706942000

$-1.854338000$

$-0.412985000$

0.176948000

4.754379000

3.349146000

4.754379000

3. 349146000

$\odot .592562000$

$-1.931719000$

$-3.804921000$

$-3.853000000$

$-3.804921000$

$-1.931719000$

$\odot .592562000$
1.406852000

$-1.217454000$

1.217454000

$-1.406852000$

3.474157000

2. 540089000

2.765529000

1. 242411000

3.339226000

0.000000000

2. 590001000

$-1.242411000$

2.968578000

$-2.540089000$

$-1.804407000$

$-2.968578000$

$-0.710901000$

$-2.590001000$

$-3.339226000$

0.710901000

$-2.765529000$

1.804407000

$-3.474157000$

0.0000000000

0.000000000

$-1.726872000$

$-3.989348000$

1.726872000

3.989348000

4.426656000

4.555866000

2. 714044000

0.000000000

$-2.714044000$

$-4.555866000$

$-4.426656000$

end

SYMMETRY C(s) 
CHARGE $\odot$

INTEGRATION

accint 6.0

End

RELATIVISTIC Scalar ZORA

GEOMETRY

Iterations 500

Converge $\mathrm{e}=1 . \mathrm{OE}-3 \mathrm{grad}=1 . \mathrm{OE}-3 \mathrm{rad}=1 . \mathrm{OE}-2$

End

SCF

Converge $1.0 \mathrm{E}-7$

Iterations 500

END

BASIS

Type ZORA/TZ2P

Core None

END

$\mathrm{XC}$

gga OLYP

END

endinput 


\section{Optimized all-electron OLYP/ZORA-STO-TZ2P Cartesian coordinates $(\AA)$.}

1. $\operatorname{Ir}[$ Cor $](C) ; C_{s} ; S=0 ;$ Total bonding energy $=-248.34690085$
$\mathrm{eV}$

\begin{tabular}{|c|c|}
\hline$C$ & 0.119235000 \\
\hline C & ๑. 119235000 \\
\hline C & $\odot .51609000$ \\
\hline C & 0.51609000 \\
\hline C & ๑.92694200 \\
\hline C & ๑. 92694200 \\
\hline C & 1.14359500 \\
\hline C & 1.14359500 \\
\hline C & 1.30834700 \\
\hline C & 1.30834700 \\
\hline C & 1.36988300 \\
\hline C & 1.36988300 \\
\hline C & 2.61024700 \\
\hline C & $-0.4369820 \odot$ \\
\hline C & -0.43698200 \\
\hline C & -0.44654200 \\
\hline C & -0.44654200 \\
\hline C & $-\odot .6598280 \odot$ \\
\hline C & $-\odot .7837790 \odot$ \\
\hline C & -0.78377900 \\
\hline $\mathrm{H}$ & 0.42944000 \\
\hline $\mathrm{H}$ & ๑ . 42944000 \\
\hline $\mathrm{H}$ & 1.09894500 \\
\hline $\mathrm{H}$ & 1.09894500 \\
\hline $\mathrm{H}$ & 1.53763200 \\
\hline $\mathrm{H}$ & 1.53763200 \\
\hline $\mathrm{H}$ & -0.59544600 \\
\hline $\mathrm{H}$ & $-\odot .5954460 \odot$ \\
\hline $\mathrm{H}$ & -1.10379700 \\
\hline $\mathrm{H}$ & -1.25117500 \\
\hline $\mathrm{H}$ & -1.25117500 \\
\hline Ir & 1.03449100 \\
\hline $\mathrm{N}$ & ๑. 12133300 \\
\hline $\mathrm{N}$ & 0.12133300 \\
\hline $\mathrm{N}$ & 1.06988100 \\
\hline $\mathrm{N}$ & 1 \\
\hline
\end{tabular}

$-0.708026000$

$-0.708026000$

0.517833000

$\odot .517833000$

1.642006000

1.642006000

3.009008000

3. 009008000

2.828301000

2.828301000

3.735480000

3. 735480000

$-0.577713000$

$-2.274649000$

$-2.274649000$

$-1.830529000$

$-1.830529000$

$-2.861843000$

$-2.782247000$

$-2.782247000$

$\odot .619520000$

$\odot .619520000$

3. 402809000

3. 402809000

4.802227000

4.802227000

$-1.880880000$

$-1.880880000$

$-3.852291000$

$-3.741709000$

$-3.741709000$

๑. 040740000

$-1.026971000$

$-1.026971000$

1. 581412000

1.581412000
2.785837000

$-2.785837000$

3. 336985000

$-3.336985000$

2. 603246000

$-2.603246000$

2. 962922000

$-2.962922000$

0.710347000

$-0.710347000$

1.799983000

$-1.799983000$

0.000000000

1.251310000

$-1.251310000$

3. 475237000

- 3.475237000

0.000000000

2.545820000

$-2.545820000$

4.414136000

$-4.414136000$

3. 970575000

$-3.970575000$

1. 727347000

$-1.727347000$

4.546825000

$-4.546825000$

0.000000000

2.728649000

$-2.728649000$

๑ . 000000000

1.434857000

$-1.434857000$

1. 243301000

$-1.243301000$

2. $\operatorname{Ir}[\operatorname{Cor}](C) ; C_{s} ; S=1 ;$ Total bonding energy $=-246.54046220$ eV

$\begin{array}{ll}\text { C } & 0.126072000 \\ \text { C } & 0.126072000 \\ \text { C } & 0.550011000 \\ \text { C } & 0.550011000\end{array}$

$$
\begin{array}{r}
-0.707152000 \\
-0.707152000 \\
\odot .49297500 \odot \\
\odot .49297500 \odot
\end{array}
$$

2.787282000

$-2.787282000$

3.344899000

$-3.344899000$ 


\begin{tabular}{|c|c|}
\hline C & ๑. 932388000 \\
\hline C & $\odot .932388000$ \\
\hline C & $1.12849900 \odot$ \\
\hline C & 1.12849900 \\
\hline C & 1.32172100 \\
\hline C & 1.321721000 \\
\hline C & 1.362721000 \\
\hline C & 1.36272100 \\
\hline C & 2.59571700 \\
\hline C & $-\odot .43217900$ \\
\hline C & -0.432179000 \\
\hline C & -0.474401000 \\
\hline C & -0.47440100 \\
\hline C & $-\odot .70934900$ \\
\hline C & $-\odot .7920240 \odot$ \\
\hline C & -0.792024000 \\
\hline $\mathrm{H}$ & $\odot .49764600$ \\
\hline $\mathrm{H}$ & $\odot .49764600$ \\
\hline $\mathrm{H}$ & 1.06725400 \\
\hline $\mathrm{H}$ & 1.06725400 \\
\hline $\mathrm{H}$ & 1.52987700 \\
\hline $\mathrm{H}$ & 1.52987700 \\
\hline $\mathrm{H}$ & $-0.5532370 \odot$ \\
\hline $\mathrm{H}$ & -0.55323700 \\
\hline $\mathrm{H}$ & -1.15198200 \\
\hline $\mathrm{H}$ & -1.25594700 \\
\hline $\mathrm{H}$ & $-1.2559470 \odot$ \\
\hline $\operatorname{Ir}$ & $1.0157550 €$ \\
\hline $\mathrm{N}$ & ๑ . 09218100 \\
\hline $\mathrm{N}$ & ๑ . 09218100 \\
\hline $\mathrm{N}$ & 1.09230200 \\
\hline $\mathrm{N}$ & 1.09 \\
\hline
\end{tabular}

1. 650423000

1.650423000

3. 022823000

3. 022823000

2. 850126000

2. 850126000

3. 754176000

3. 754176000

$-0.584478000$

$-1.850100000$

$-1.850100000$

$-2.268286000$

$-2.268286000$

$-2.852876000$

$-2.784882000$

$-2.784882000$

$\odot .586447000$

$\odot .586447000$

3. 412630000

3. 412630000

4.820945000

4.820945000

$-1.910558000$

$-1.910558000$

$-3.843823000$

$-3.746138000$

$-3.746138000$

$\odot .038030000$

$-1.014683000$

$-1.014683000$

1.580861000

1.580861000
2.591980000

$-2.591980000$

2. 958864000

$-2.958864000$

$\odot .707889000$

$-0.707889000$

1.795429000

$-1.795429000$

๑ . $0000000 \odot \odot$

3. 481974000

$-3.481974000$

1. 249040000

$-1.249040000$

๑. . $0000000 \odot \odot$

2.553056000

$-2.553056000$

4.423849000

$-4.423849000$

3.966681000

- 3.966681000

1.725290000

$-1.725290000$

4.556219000

$-4.556219000$

๑. . 000000000

2. 736555000

$-2.736555000$

$0.00000000 \odot$

1. 428282000

$-1.428282000$

1. 249885000

$-1.249885000$

\section{3. $\{\operatorname{Ir}[\operatorname{Cor}](C)\}^{-} ; C_{s} ; S=1 / 2 ;$ Total bonding energy $=-$} $249.50413800 \mathrm{eV}$

$\begin{array}{lr}\text { C } & 0.117682000 \\ \text { C } & 0.117682000 \\ \text { C } & 0.547707000 \\ \text { C } & 0.547707000 \\ \text { C } & 0.924941000 \\ \text { C } & 0.924941000 \\ \text { C } & 1.158519000 \\ \text { C } & 1.15851900 \odot \\ \text { C } & 1.280604000 \\ \text { C } & 1.280604000 \\ \text { C } & 1.371822000 \\ \text { C } & 1.371822000 \\ \text { C } & 2.56514900 \odot \\ \text { C } & -0.424164000\end{array}$
$-0.710317000$
$-0.710317000$
$\odot .501774000$
0.501774000
1.656121000
1.656121000
3.007755000
3.007755000
2.858106000
2.858106000
3.750611000
3.750611000
$-\odot .546135000$
$-1.848559000$

2.797417000

$-2.797417000$

3. 355296000

$-3.355296000$

2. 618590000

$-2.618590000$

2. 983521000

$-2.983521000$

$\odot .719287000$

- $\odot .719287000$

1.800638000

$-1.800638000$

๑ . 000000000

3.487465000 


\begin{tabular}{|c|c|}
\hline C & -0.424164000 \\
\hline C & -0.475659000 \\
\hline C & $-\odot .475659 \odot \odot$ \\
\hline C & -0.70231900 \\
\hline C & -0.78388100 \\
\hline C & -0.783881000 \\
\hline $\mathrm{H}$ & $\odot .501343000$ \\
\hline $\mathrm{H}$ & 0.50134300 \\
\hline $\mathrm{H}$ & 1.12561400 \\
\hline $\mathrm{H}$ & 1.125614000 \\
\hline $\mathrm{H}$ & 1.55184700 \\
\hline $\mathrm{H}$ & 1.55184700 \\
\hline $\mathrm{H}$ & -0.54571200 \\
\hline $\mathrm{H}$ & $-\odot .5457120 \odot$ \\
\hline $\mathrm{H}$ & -1.13703700 \\
\hline $\mathrm{H}$ & -1.24097800 \\
\hline $\mathrm{H}$ & -1.24097800 \\
\hline $\operatorname{Ir}$ & ๑. 97868200 \\
\hline $\mathrm{N}$ & 0.07657300 \\
\hline $\mathrm{N}$ & 0.07657300 \\
\hline $\mathrm{N}$ & 1.02945900 \\
\hline $\mathrm{N}$ & 1.029 \\
\hline
\end{tabular}

$-1.848559000$

$-2.276157000$

$-2.276157000$

$-2.858548000$

$-2.794994000$

$-2.794994000$

0.585736000

$\odot .585736000$

3. 399817000

3. 399817000

4.816783000

4.816783000

$-1.912673000$

$-1.912673000$

$-3.854528000$

$-3.760600000$

$-3.760600000$

0.061573000

$-1.012563000$

$-1.012563000$

1. 606013000

1.606013000
$-3.487465000$

1.254713000

$-1.254713000$

0.000000000

2. 548589000

$-2.548589000$

4.436952000

$-4.436952000$

3.993322000

$-3.993322000$

1. 732458000

$-1.732458000$

4.562641000

$-4.562641000$

0.000000000

2. 733557000

$-2.733557000$

0.000000000

1.439503000

$-1.439503000$

1.250766000

$-1.250766000$

4. $\operatorname{Ir}[\mathrm{Cor}](\mathrm{NMe}) 2 ; C_{2 v} ; S=0 ;$ Total bonding energy = 292.95974054 eV

\begin{tabular}{|c|c|}
\hline C & 0.000000000 \\
\hline C & $\odot .0000 \odot \odot ० ० \odot$ \\
\hline C & $\odot .0 \odot \odot ० \odot \odot \odot ० \odot$ \\
\hline C & 0.71995600 \\
\hline C & 1.27394100 \\
\hline $\mathrm{C}$ & 1.844469000 \\
\hline C & 2.590010000 \\
\hline C & 2.61397300 \\
\hline C & 2.80341000 \\
\hline C & 3.000724000 \\
\hline C & 3.32824600 \\
\hline C & 3.51476500 \\
\hline C & $-\odot .7199560 \odot$ \\
\hline C & -1.27394100 \\
\hline C & -1.84446900 \\
\hline C & -2.59001000 \\
\hline C & -2.61397300 \\
\hline C & -2.80341000 \\
\hline C & -3.00072400 \\
\hline C & -3.32824600 \\
\hline C & $-3.5147650 \odot$ \\
\hline $\mathrm{H}$ & $0.0000000 \odot$ \\
\hline $\mathrm{H}$ & $\odot .0000 \odot \odot \odot \odot$ \\
\hline $\mathrm{H}$ & $\odot . \odot \odot \odot \odot \odot \odot \odot ८$ \\
\hline
\end{tabular}
0.000000000
2.849371000
$-2.849371000$
๑. 000000000
$\odot .00000 \odot \odot \odot \odot$
$\odot .000000000$
0.000000000
$\odot .000000000$
$\odot .00000000 \odot$
๑. . 000000000
๑. 000000000
$\odot .00000 \odot \odot \odot \odot$
๑. . $00 \odot \odot ० ० \odot \odot \odot$
०. 000000000
$\odot .000000000$
0.000000000
0.000000000
०. 000000000
๑. 000000000
$\odot .00000000 \odot$
$\odot .000000000$
$\odot .0000000 \odot \odot$
3. 811923000
$-3.811923000$

$-3.114442000$

$-0.859809000$

$-0.859809000$

3.010491000

$-2.496768000$

3.892067000

- 3.091448000

1.726694000

$-0.813644000$

3. 112574000

0.503999000

- 2.070597000

3. 010491000

$-2.496768000$

3.892067000

$-3.091448000$

1. 726694000

$-0.813644000$

3. 112574000

0.503999000

$-2.070597000$

-4 . 200885000

$-0.325751000$

$-0.325751000$ 


\begin{tabular}{|c|c|}
\hline $\mathrm{H}$ & ๑.8907090०९ \\
\hline $\mathrm{H}$ & ๑.890709000 \\
\hline $\mathrm{H}$ & 1.805074000 \\
\hline $\mathrm{H}$ & 2.795248000 \\
\hline $\mathrm{H}$ & 4.018817000 \\
\hline $\mathrm{H}$ & 4.412348000 \\
\hline $\mathrm{H}$ & 4.593296000 \\
\hline $\mathrm{H}$ & -0.890709000 \\
\hline $\mathrm{H}$ & -0.890709000 \\
\hline $\mathrm{H}$ & -1.805074000 \\
\hline $\mathrm{H}$ & -2.795248000 \\
\hline $\mathrm{H}$ & -4.018817000 \\
\hline $\mathrm{H}$ & -4.412348000 \\
\hline $\mathrm{H}$ & -4.593296000 \\
\hline Ir & 0.000000000 \\
\hline $\mathrm{N}$ & $\odot .0 \odot \odot \odot \odot \odot \odot \odot \odot$ \\
\hline $\mathrm{N}$ & $\odot .0 \odot \odot \odot \odot \odot \odot \odot \odot ~$ \\
\hline $\mathrm{N}$ & 1.250579000 \\
\hline $\mathrm{N}$ & 1.469582000 \\
\hline $\mathrm{N}$ & -1.250579000 \\
\hline . & -1.469582000 \\
\hline
\end{tabular}

2.824070000

$-2.824070000$

0.000000000

๑ . 000000000

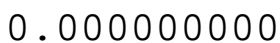

○. 000000000

$\odot .000000000$

2.824070000

$-2.824070000$

๑. . 000000000

०. 000000000

๑. 000000000

๑. . 000000000

๑. $0000000 \odot \odot$

$\odot .00000000 \odot$

1. 806491000

$-1.806491000$

०. $00000000 \odot$

๑. . 000000000

$\odot .000000000$

๑ . 000000000
$-1.508976000$

$-1.508976000$

4.974057000

$-4.155162000$

3. 482187000

$\odot .574813000$

$-2.173438000$

$-1.508976000$

$-1.508976000$

4.974057000

$-4.155162000$

3.482187000

$\odot .574813000$

$-2.173438000$

$\odot .245715000$

$\odot .079446000$

0.079446000

1.745928000

$-1.143475000$

1.745928000

$-1.143475000$

5. $\operatorname{Ir}[\mathrm{Cor}](\mathrm{NMe})_{2} ; C_{2 v} ; S=1 ;$ Total bonding energy = $292.10392868 \mathrm{eV}$

\begin{tabular}{|c|c|}
\hline C & 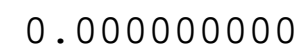 \\
\hline 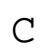 & 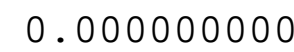 \\
\hline C & ०.00000000० \\
\hline C & ๑. 71927300 \\
\hline $\mathrm{C}$ & 1.26736300 \\
\hline C & 1.83702100 \\
\hline C & 2.5837320 \\
\hline C & 2.61085200 \\
\hline C & 2.79782500 \\
\hline $\mathrm{C}$ & 2.994194000 \\
\hline C & 3. 32893800 \\
\hline $\mathrm{C}$ & 3.50849500 \\
\hline C & -0.71927300 \\
\hline C & -1.26736300 \\
\hline C & -1.83702100 \\
\hline C & -2.58373200 \\
\hline $\mathrm{C}$ & -2.61085200 \\
\hline $\mathrm{C}$ & -2.79782500 \\
\hline $\mathrm{C}$ & -2.99419400 \\
\hline C & -3.32893800 \\
\hline $\mathrm{C}$ & -3.50849500 \\
\hline $\mathrm{H}$ & 0.00000000 \\
\hline $\mathrm{H}$ & $\odot .0000000 \odot$ \\
\hline $\mathrm{H}$ & ० . 00000000 \\
\hline $\mathrm{H}$ & 0.87522600 \\
\hline
\end{tabular}
0.000000000
2.908355000
$-2.908355000$
0.000000000
$\odot .000000000$
$\odot .00000000 \odot$
$\odot .00000000 \odot$
0.000000000
$\odot .0000000 \odot \odot$
$\odot .000000 \odot \odot \odot$
$\odot .000000000$
$\odot .000000000$
๑. $.000 \odot \odot ० ० ० \odot$
$\odot .00000 \odot \odot \odot \odot$
०. 000000000
$\odot .00000000 \odot$
$\odot .0000 \odot \odot \odot ० \odot$
$\odot .000000000$
$\odot .000000000$
0.000000000
๑. . $00000000 \odot$
○. 000000000
2. 539856000
$-2.539856000$
3. 566189000

$-3.553664000$

0.785392000

0.785392000

2.558477000

$-2.932757000$

3. 447065000

$-3.528042000$

1. 280928000

$-1.253578000$

2.670620000

๑. 053862000

$-2.510491000$

2. 558477000

$-2.932757000$

3.447065000

$-3.528042000$

1. 280928000

$-1.253578000$

2.670620000

0.053862000

$-2.510491000$

$-4.639807000$

1.819817000

1. 819817000

$\odot .637753000$ 


\begin{tabular}{|c|c|}
\hline $\mathrm{H}$ & ๑. $87522600 \odot$ \\
\hline $\mathrm{H}$ & $1.79198300 \odot$ \\
\hline $\mathrm{H}$ & 2.78651400 \\
\hline $\mathrm{H}$ & 4.01142600 \\
\hline $\mathrm{H}$ & 4.41320200 \\
\hline $\mathrm{H}$ & 4.58685900 \\
\hline $\mathrm{H}$ & $-0.8752260 \odot$ \\
\hline $\mathrm{H}$ & $-\odot .8752260 \odot$ \\
\hline $\mathrm{H}$ & -1.79198300 \\
\hline $\mathrm{H}$ & -2.78651400 \\
\hline $\mathrm{H}$ & -4.01142600 \\
\hline $\mathrm{H}$ & -4.41320200 \\
\hline $\mathrm{H}$ & -4.58685900 \\
\hline $\operatorname{Ir}$ & ๑ . ๑००००००८ \\
\hline $\mathrm{N}$ & ๑. . $0000000 \odot$ \\
\hline $\mathrm{N}$ & 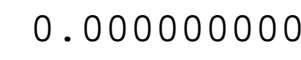 \\
\hline $\mathrm{N}$ & 1.25432400 \\
\hline $\mathrm{N}$ & 1.45444600 \\
\hline $\mathrm{N}$ & -1.25432400 \\
\hline $\mathrm{N}$ & -1.45444600 \\
\hline
\end{tabular}

$-3.566189000$

0.000000000

○. 000000000

๑ . 000000000

० . 000000000

0.000000000

3. 566189000

$-3.566189000$

๑. . $0000000 \odot \odot$

๑. . $0000000 \odot \odot$

๑ . $0000000 \odot \odot$

๑. 000000000

๑. . 000000000

๑ . $00000 \odot \odot \odot \odot$

1.937230000

$-1.937230000$

0.000000000

○. 000000000

$\odot .000000000$

$\odot .000000000$
0.637753000

4.528586000

$-4.592087000$

3.042831000

$\odot .122294000$

$-2.612906000$

0.637753000

$\odot .637753000$

4.528586000

$-4.592087000$

3. 042831000

$\odot .122294000$

$-2.612906000$

$-0.223974000$

$-0.234284000$

- 0.234284000

1.286754000

$-1.579857000$

1.286754000

$-1.579857000$

6. $\left\{\operatorname{Ir}[\mathrm{Cor}](\mathrm{NMe})_{2}\right\}^{-}$; $294.71565569 \mathrm{eV}$

\begin{tabular}{|c|c|}
\hline C & $\odot . \odot \odot \odot \odot \odot \odot ० ० \odot$ \\
\hline C & $\odot . ๑ \odot \odot \odot \odot \odot \odot \odot \odot$ \\
\hline c & $\odot . ๑ \odot \odot \odot \odot \odot ० ० \odot$ \\
\hline c & $\odot .724083000$ \\
\hline C & 1.270556000 \\
\hline C & 1.845436000 \\
\hline C & 2.588948000 \\
\hline C & 2.607679000 \\
\hline C & 2.792377000 \\
\hline C & 3.001732000 \\
\hline C & 3.319509000 \\
\hline C & 3.510877000 \\
\hline C & -0.724083000 \\
\hline C & -1.270556000 \\
\hline C & -1.845436000 \\
\hline C & -2.588948000 \\
\hline C & $-2.60767900 \odot$ \\
\hline C & -2.792377000 \\
\hline C & -3.001732000 \\
\hline C & $-3.31950900 \odot$ \\
\hline C & -3.510877000 \\
\hline $\mathrm{H}$ & $\odot . \odot \odot \odot \odot \odot \odot \odot \odot \odot$ \\
\hline 11 & $\odot .0 \odot \odot \odot \odot \odot \odot \odot \odot ~$ \\
\hline $\mathrm{H}$ & $\odot . \diamond \odot \odot \odot \odot \odot \odot \diamond \diamond$ \\
\hline $\mathrm{H}$ & $\odot .882293000$ \\
\hline & \\
\hline
\end{tabular}
0.000000000
2. 914803000
$-2.914803000$
0.000000000
0.000000000
0.000000000
$\odot .000000000$
$\odot .0000000000$
0.000000000
$\odot .0000000000$
0.0000000000
$\odot .0000000000$
0.0000000000
0.0000000000
0.0000000000
$\odot .000000000$
$\odot .0000000000$
0.000000000
0.000000000
0.000000000
$\odot .000000000$
0.000000000
2.576336000
$-2.576336000$
3.570437000
$-3.570437000$

$-3.551777000$

0.741947000

0.741947000

2.573187000

$-2.930550000$

3.459730000

$-3.528354000$

1. 293407000

$-1.251429000$

2.678272000

0.062116000

$-2.507175000$

2. 573187000

$-2.930550000$

3. 459730000

$-3.528354000$

1.293407000

$-1.251429000$

2.678272000

0.062116000

$-2.507175000$

$-4.638804000$

1.791872000

1.791872000

0.611887000

$\odot .611887000$ 


\begin{tabular}{|c|c|}
\hline $\mathrm{H}$ & 1.804987000 \\
\hline $\mathrm{H}$ & $2.79456900 \odot$ \\
\hline $\mathrm{H}$ & 4.02123300 \\
\hline $\mathrm{H}$ & 4.40494600 \\
\hline $\mathrm{H}$ & 4.590825000 \\
\hline $\mathrm{H}$ & $-\odot .88229300$ \\
\hline $\mathrm{H}$ & $-\odot .8822930 \odot$ \\
\hline $\mathrm{H}$ & -1.80498700 \\
\hline $\mathrm{H}$ & -2.79456900 \\
\hline $\mathrm{H}$ & -4.02123300 \\
\hline $\mathrm{H}$ & -4.40494600 \\
\hline $\mathrm{H}$ & $-4.5908250 \odot$ \\
\hline $\operatorname{Ir}$ & ๑. . 00000000 \\
\hline $\mathrm{N}$ & $\odot . ๑ \odot \odot \odot \odot \odot \odot \odot$ \\
\hline $\mathrm{N}$ & 0.00000000 \\
\hline $\mathrm{N}$ & 1.24937000 \\
\hline $\mathrm{N}$ & 1.45867900 \\
\hline $\mathrm{N}$ & -1.24937000 \\
\hline $\mathrm{N}$ & -1.45867900 \\
\hline
\end{tabular}
0.000000000
4.542790000
0.000000000
$-4.593084000$
๑. . 000000000
3.047061000
๑. . 000000000
0.130187000
๑ . $00000000 \odot$
$-2.606719000$
3. 570437000
0.611887000
$-3.570437000$
$\odot .611887000$
๑. . 000000000
4.542790000
๑ . $0000000 \odot \odot$
$-4.593084000$
๑ . $000000 \odot ० \odot$
3. 047061000
๑. 000000000
$\odot .130187000$
๑. 000000000
$-2.606719000$
๑. 000000000
$-0.210307000$
1.890387000
$-0.235103000$
$-1.890387000$
$-0.235103000$
๑. $00000000 \odot$
1.310550000
0.000000000
$-1.582629000$
๑. . 000000000
1.310550000
०. 000000000
$-1.582629000$

\section{7. $\operatorname{Re}[$ Cor $](C) ; C_{s} ; S=0 ;$ Total bonding energy $=-249.53386250$ eV}

\begin{tabular}{|c|c|}
\hline C & $\odot .3653920 \odot \odot$ \\
\hline C & $\odot .365392000$ \\
\hline c & $\odot .763326000$ \\
\hline C & $\odot .76332600 \odot$ \\
\hline C & $1.02419200 \odot$ \\
\hline c & 1.024192000 \\
\hline C & $1.07315900 \odot$ \\
\hline C & 1.073159000 \\
\hline C & 1.149925000 \\
\hline C & 1.149925000 \\
\hline c & 1.168961000 \\
\hline C & 1.168961000 \\
\hline C & 2.849144000 \\
\hline c & -0.364461000 \\
\hline C & -0.364461000 \\
\hline C & -0.498165000 \\
\hline C & $-\odot .49816500 \odot$ \\
\hline C & $-\odot .82047600 \odot$ \\
\hline C & -0.901428000 \\
\hline C & -0.901428000 \\
\hline $\mathrm{H}$ & $\odot .70529100 \odot$ \\
\hline $\mathrm{H}$ & $\odot .705291000$ \\
\hline H & $1.20442700 \odot$ \\
\hline 7 & $1.20442700 \odot$ \\
\hline t & 1.23683306 \\
\hline & 1.236833000 \\
\hline & -0.517163 \\
\hline
\end{tabular}
$-0.828265000$
$-0.828265000$
0.430222000
0.430222000
1.585928000
1.585928000
2.827301000
2.827301000
2.965514000
2.965514000
3.728971000
3. 728971000
$-0.303859000$
$-1.864854000$
$-1.864854000$
$-2.209337000$
$-2.209337000$
$-2.716523000$
$-2.694034000$
$-2.694034000$
0.552888000
๑. 552888000
3. 331585000
3. 331585000
4.807164000
4.807164000
$-1.921843000$

2.844286000

$-2.844286000$

3. 348574000

$-3.348574000$

2. 592328000

$-2.592328000$

0.716320000

$-0.716320000$

2.972785000

$-2.972785000$

1. 816688000

$-1.816688000$

๑. 000000000

3. 517295000

$-3.517295000$

1. 271898000

$-1.271898000$

0.000000000

2. 561572000

$-2.561572000$

4.426591000

$-4.426591000$

3.990675000

- 3.990675000

1.753752000

$-1.753752000$

4.587922000 


$\begin{array}{lr}\mathrm{H} & -0.517163000 \\ \mathrm{H} & -1.437229000 \\ \mathrm{H} & -1.548229000 \\ \mathrm{H} & -1.548229000 \\ \mathrm{~N} & 0.329228000 \\ \mathrm{~N} & 0.329228000 \\ \mathrm{~N} & 1.004972000 \\ \mathrm{~N} & 1.004972000 \\ \mathrm{Re} & 1.151942000\end{array}$

$-1.921843000$

$-3.610960000$

$-3.546799000$

- 3.546799000

$-1.113695000$

$-1.113695000$

1.564478000

1.564478000

- 0.063044000
$-4.587922000$

0.000000000

2.725907000

$-2.725907000$

1. 496093000

$-1.496093000$

1. 218772000

$-1.218772000$

๑ . $0000000 \odot \odot$

8. $\operatorname{Re}[$ Cor $](C) ; C_{s} ; S=1 ;$ Total bonding energy $=-249.70563716$ eV

\begin{tabular}{|c|c|}
\hline $\mathcal{C}$ & 0.163717000 \\
\hline C & ๑. 163717000 \\
\hline C & 0.552409000 \\
\hline C & 0.552409000 \\
\hline C & ๑. 98726700 \\
\hline C & ๑. . 98726700 \\
\hline C & 1.10386100 \\
\hline C & 1.103861000 \\
\hline C & 1.31755300 \\
\hline C & 1.31755300 \\
\hline C & 1.35341700 \\
\hline C & 1.35341700 \\
\hline C & 2.83038500 \\
\hline C & -0.39908000 \\
\hline C & $-\odot .39908000$ \\
\hline C & $-\odot .4852560 \odot$ \\
\hline C & $-\odot .4852560 \odot$ \\
\hline C & -0.64501900 \\
\hline C & $-\odot .8300750 \odot$ \\
\hline C & -0.83007500 \\
\hline $\mathrm{H}$ & ๑. 39811900 \\
\hline $\mathrm{H}$ & ๑. 39811900 \\
\hline $\mathrm{H}$ & ๑. 99124400 \\
\hline $\mathrm{H}$ & 0.99124400 \\
\hline $\mathrm{H}$ & 1.41094800 \\
\hline $\mathrm{H}$ & 1.41094800 \\
\hline $\mathrm{H}$ & $-\odot .68997900$ \\
\hline $\mathrm{H}$ & $-\odot .6899790 \odot$ \\
\hline $\mathrm{H}$ & -1.16325900 \\
\hline $\mathrm{H}$ & -1.36058600 \\
\hline $\mathrm{H}$ & -1.36058600 \\
\hline $\mathrm{N}$ & ๑. 23132200 \\
\hline $\mathrm{N}$ & $\odot .2313220 €$ \\
\hline $\mathrm{N}$ & 1.19933600 \\
\hline $\mathrm{N}$ & 1.19933600 \\
\hline $\mathrm{Re}$ & 1.225365 \\
\hline
\end{tabular}

$-0.736965000$

$-0.736965000$

$\odot .518174000$

0.518174000

1.632179000

1.632179000

3. 019464000

3. 019464000

3.739553000

3.739553000

2. 801895000

2. 801895000

$-0.647124000$

$-2.284162000$

$-2.284162000$

$-1.819028000$

$-1.819028000$

$-2.842424000$

$-2.764838000$

$-2.764838000$

0.653578000

$\odot .653578000$

3. 422324000

3. 422324000

4.814358000

4.814358000

$-1.847081000$

$-1.847081000$

$-3.797496000$

$-3.692996000$

$-3.692996000$

$-1.073195000$

$-1.073195000$

1.556580000

1.556580000

$-0.056573000$
2.801696000

- 2.801696000

3. 331222000

$-3.331222000$

2. 601879000

$-2.601879000$

2. 958173000

$-2.958173000$

1.795797000

$-1.795797000$

0.719825000

$-0.719825000$

0.000000000

1.270036000

$-1.270036000$

3.487382000

$-3.487382000$

0.000000000

2.552023000

$-2.552023000$

4. 398119000

$-4.398119000$

3. 956920000

$-3.956920000$

1. 707351000

$-1.707351000$

4.550341000

$-4.550341000$

0.000000000

2. 724121000

$-2.724121000$

1. 469117000

$-1.469117000$

1. 245504000

$-1.245504000$

$\odot .000000000$ 
9. $\{\operatorname{Re}[\text { Cor }](C)\}^{-} ; C_{s} ; S=1 / 2 ;$ Total bonding energy $=-$ $252.36158143 \mathrm{eV}$

\begin{tabular}{|c|c|c|c|}
\hline C & $\odot .19062700 \odot$ & $-0.74253100 \odot$ & $2.8074540 \mathrm{C}$ \\
\hline C & $\odot .19062700 \odot$ & -0.742531000 & -2.80745400 \\
\hline C & 0.573144000 & 0.505643000 & 3.33560506 \\
\hline C & $\odot .573144000$ & 0.505643000 & -3.3356050 \\
\hline C & $1.0212450 \odot \odot$ & $1.62754600 \odot$ & 2.6016150 \\
\hline C & $1.02124500 \odot$ & 1.627546000 & -2.6016150 \\
\hline C & 1.077442000 & $3.02028900 \odot$ & 2.9507560 \\
\hline C & $1.07744200 \odot$ & $3.02028900 \odot$ & -2.9507560 \\
\hline C & 1. $29919300 \odot$ & $3.74286200 \odot$ & 1.7821200 \\
\hline C & 1.299193000 & 3.742862000 & -1.7821200 \\
\hline C & $1.39896000 \odot$ & $2.80125700 \odot$ & $\odot .7164570$ \\
\hline C & 1.398960000 & 2.801257000 & -0.7164570 \\
\hline C & $2.8862300 \odot \odot$ & $-\odot .67891300 \odot$ & $\odot . \odot \odot \odot \odot \odot \odot \odot$ \\
\hline C & $-\odot .3746860 \odot \odot$ & -2.294177000 & 1.2683930 \\
\hline C & $-\odot .3746860 \odot \odot$ & -2.294177000 & -1.2683930 \\
\hline C & $-\odot .5064280 \odot \odot$ & $-1.8051600 \odot \odot$ & 3.4863410 \\
\hline C & $-\odot .50642800 \odot$ & $-1.80516000 \odot$ & -3.4863410 \\
\hline C & $-\odot .62472800 \odot$ & -2.858213000 & 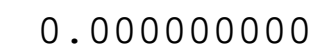 \\
\hline C & $-\odot .8520940 \odot \odot$ & -2.751712000 & 2.5497670 \\
\hline C & $-\odot .85209400 \odot$ & -2.751712000 & -2.5497670 \\
\hline $\mathrm{H}$ & $\odot .38660500 \odot$ & $\odot .65289000 \odot$ & 4.3964270 \\
\hline $\mathrm{H}$ & $\odot .38660500 \odot$ & $\odot .65289000 \odot$ & -4.3964270 \\
\hline $\mathrm{H}$ & ๑.910786000 & 3.428236000 & $3.9412350 c$ \\
\hline $\mathrm{H}$ & $\odot .91078600 \odot$ & 3.428236000 & -3.9412350 \\
\hline $\mathrm{H}$ & 1.347895000 & 4.821296000 & 1.6873350 \\
\hline $\mathrm{H}$ & 1.347895000 & 4.821296000 & -1.6873350 \\
\hline $\mathrm{H}$ & $-\odot .7466 \odot 4 \odot \odot \odot$ & $-1.81354400 \odot$ & 4.5436360 \\
\hline $\mathrm{H}$ & $-\odot .7466 \odot 4 \odot \odot \odot$ & $-1.81354400 \odot$ & -4.5436360 \\
\hline $\mathrm{H}$ & -1.170114000 & -3.798482000 & $0.0000000 \mathrm{C}$ \\
\hline $\mathrm{H}$ & $-1.41976600 \odot$ & -3.660461000 & 2.7154830 \\
\hline $\mathrm{H}$ & -1.419766000 & -3.660461000 & -2.7154830 \\
\hline $\mathrm{N}$ & $\odot .2926420 \odot \odot$ & $-1.10915800 \odot$ & 1.4781150 \\
\hline $\mathrm{N}$ & $\odot .29264200 \odot$ & -1.109158000 & -1.4781150 \\
\hline $\mathrm{N}$ & 1.285545000 & 1.547362000 & 1.2599870 \\
\hline $\mathrm{N}$ & $1.28554500 \odot$ & 1.547362000 & -1.2599870 \\
\hline $\operatorname{Re}$ & $1.27550600 \odot$ & $-\odot .06960700 \odot$ & $\odot .0 \odot \odot \odot \odot \odot \odot$ \\
\hline
\end{tabular}

10. $\operatorname{Re}[\mathrm{Cor}](\mathrm{NMe})_{2} ; C_{2 v} ; S=0 ;$ Total bonding energy $=-$ $296.38964416 \mathrm{eV}$

\begin{tabular}{|c|c|c|c|}
\hline$C$ & $\odot . \diamond \odot \odot \odot \odot \odot \odot \odot \odot$ & $\odot . ๑ \odot \odot \odot \odot \odot \odot \odot \odot$ & -2.9796660 \\
\hline C & $\odot . ๑ \odot \odot \odot \odot \odot \odot \odot \odot ~$ & 2.968988000 & -0.9432050 \\
\hline C & 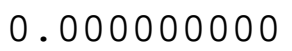 & -2.968988000 & -0.9432050 \\
\hline C & $\odot .71732200 \odot$ & $\odot .000000000$ & 3.0069620 \\
\hline C & 1. $3161800 \odot \odot$ & 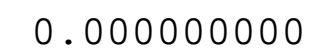 & -2.411876 \\
\hline 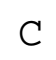 & 1.828335000 & 0.006 & 3.893327 \\
\hline C & 2.613 & 0.000000000 & 1.72966 \\
\hline
\end{tabular}




\begin{tabular}{|c|c|}
\hline $\mathrm{C}$ & 2.619543000 \\
\hline C & 2.934263000 \\
\hline $\mathrm{C}$ & 2.984856000 \\
\hline C & 3.402875000 \\
\hline C & 3.592799000 \\
\hline $\mathrm{C}$ & -0.717322000 \\
\hline $\mathrm{C}$ & -1.316180000 \\
\hline $\mathrm{C}$ & -1.828335000 \\
\hline C & - 2.613371000 \\
\hline C & -2.619543000 \\
\hline C & -2.934263000 \\
\hline C & -2.984856000 \\
\hline $\mathrm{C}$ & -3.402875000 \\
\hline C & -3.592799000 \\
\hline $\mathrm{H}$ & ๑. . 000000000 \\
\hline $\mathrm{H}$ & $\odot . ๑ \odot \odot \odot \odot \odot \odot \odot \odot$ \\
\hline $\mathrm{H}$ & ๑. 000000000 \\
\hline $\mathrm{H}$ & ๑. 895418000 \\
\hline $\mathrm{H}$ & ๑. 895418000 \\
\hline $\mathrm{H}$ & 1.782145000 \\
\hline $\mathrm{H}$ & 2.784058000 \\
\hline $\mathrm{H}$ & 4.003163000 \\
\hline $\mathrm{H}$ & 4.479464000 \\
\hline $\mathrm{H}$ & 4.664375000 \\
\hline $\mathrm{H}$ & -0.895418000 \\
\hline $\mathrm{H}$ & -0.895418000 \\
\hline $\mathrm{H}$ & -1.782145000 \\
\hline $\mathrm{H}$ & -2.784058000 \\
\hline $\mathrm{H}$ & -4.003163000 \\
\hline $\mathrm{H}$ & -4.479464000 \\
\hline $\mathrm{H}$ & -4.664375000 \\
\hline $\mathrm{N}$ & 0.000000000 \\
\hline $\mathrm{N}$ & ๑. 000000000 \\
\hline $\mathrm{N}$ & 1. 232349000 \\
\hline $\mathrm{N}$ & 1.608137000 \\
\hline $\mathrm{N}$ & -1.232349000 \\
\hline $\mathrm{N}$ & -1.608137000 \\
\hline $\mathrm{Re}$ & 0.000000000 \\
\hline
\end{tabular}

๑. . 000000000

$\odot .00000000 \odot$

๑. $00000000 \odot$

๑ . 000000000

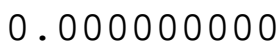

๑. . 000000000

๑. . 000000000

$\odot .000000000$

๑ . $0000000 \odot \odot$

๑. . 000000000

०. 000000000

๑. 000000000

๑. . 000000000

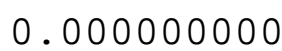

0.000000000

3.788245000

$-3.788245000$

3. 095215000

$-3.095215000$

๑ . $0000000 \odot \odot$

๑ . $00000000 \%$

๑. $00000000 \odot$

$\odot .000000000$

๑ . $0000000 \odot \odot$

3. 095215000

$-3.095215000$

๑. . $00 \odot \odot ० ० ० ० \odot$

๑. . $0000000 \odot \odot$

๑. . $000 \odot ० ० ० ० \odot$

0.000000000

○. $00000000 \odot$

1.747389000

$-1.747389000$

0.000000000

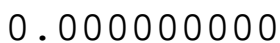

$\odot .000000 \odot \odot \odot$

$\odot .000000000$

๑ . 000000000
$-3.055032000$

$-0.785917000$

3.116471000

$\odot .549404000$

$-2.077180000$

3. 006962000

$-2.411876000$

3. 893327000

1. 729668000

$-3.055032000$

$-0.785917000$

3.116471000

๑. 549404000

- 2.077180000

$-4.067178000$

$-0.206117000$

$-0.206117000$

$-1.573177000$

$-1.573177000$

4.974931000

$-4.126220000$

3.486472000

$\odot .692386000$

$-2.235775000$

$-1.573177000$

$-1.573177000$

4.974931000

$-4.126220000$

3.486472000

0.692386000

$-2.235775000$

$-0.260437000$

$-0.260437000$

1.733695000

$-1.068065000$

1.733695000

$-1.068065000$

$\odot .151383000$

11. $\operatorname{Re}[\mathrm{Cor}](\mathrm{NMe}) 2 ; C_{2 v} ; S=1 ;$ Total bonding energy = 295.84740414 eV (non Aufbau solution)

$\begin{array}{ll}\text { C } & 0.000000000 \\ \text { C } & 0.000000000 \\ \text { C } & 0.000000000 \\ \text { C } & 0.721116000 \\ \text { C } & 1.301032000 \\ \text { C } & 1.838036000 \\ \text { C } & 2.612777000 \\ \text { C } & 2.618732000 \\ \text { C } & 2.875954000\end{array}$

0.000000000

2.952575000

$-2.952575000$

0.000000000

$\odot .00000000 \odot$

$\odot .000000000$

$\odot .00000000 \odot$

$\odot .000000000$

$\odot .00000000 \odot$
$-3.049610000$

$-\odot .896992000$

$-0.896992000$

3.021532000

$-2.460666000$

3.903557000

- 3.076395000

1.739917000

$-0.801544000$ 


\begin{tabular}{|c|c|}
\hline C & $2.99612700 \odot$ \\
\hline C & $3.36693400 \odot$ \\
\hline C & 3.56095600 \\
\hline C & -0.72111600 \\
\hline C & -1.301032000 \\
\hline C & -1.838036000 \\
\hline $\mathrm{C}$ & -2.61277700 \\
\hline C & -2.61873200 \\
\hline C & -2.87595400 \\
\hline C & -2.996127000 \\
\hline C & -3.366934000 \\
\hline C & -3.56095600 \\
\hline $\mathrm{H}$ & $\odot .0 \odot \odot \odot ० \odot \odot \odot$ \\
\hline $\mathrm{H}$ & $\odot .0 \odot \odot \odot \odot \odot \odot \odot \odot$ \\
\hline $\mathrm{H}$ & 0.00000000 \\
\hline $\mathrm{H}$ & $\odot .88237300$ \\
\hline $\mathrm{H}$ & 0.88237300 \\
\hline $\mathrm{H}$ & 1.79801800 \\
\hline $\mathrm{H}$ & $2.80255200 \odot$ \\
\hline $\mathrm{H}$ & 4.01287900 \\
\hline $\mathrm{H}$ & 4.44812600 \\
\hline $\mathrm{H}$ & 4.63621500 \\
\hline $\mathrm{H}$ & -0.882373000 \\
\hline $\mathrm{H}$ & $-\odot .88237300$ \\
\hline $\mathrm{H}$ & -1.79801800 \\
\hline $\mathrm{H}$ & -2.80255200 \\
\hline $\mathrm{H}$ & -4.01287900 \\
\hline $\mathrm{H}$ & -4.44812600 \\
\hline $\mathrm{H}$ & -4.63621500 \\
\hline $\mathrm{N}$ & 0.00000000 \\
\hline $\mathrm{N}$ & 0.00000000 \\
\hline $\mathrm{N}$ & 1.24704200 \\
\hline $\mathrm{N}$ & 1.54562500 \\
\hline $\mathrm{N}$ & -1.24704200 \\
\hline $\mathrm{N}$ & -1.54562500 \\
\hline $\mathrm{Re}$ & 0.00000000 \\
\hline
\end{tabular}

0.0000000000

3.125695000

0.000000000

0.529891000

๑. 0000000000

$-2.075624000$

$\odot .000000000$

3. 021532000

0.000000000

$-2.460666000$

0.000000000

3.903557000

$\odot .0000000000$

$-3.076395000$

0.000000000

1.739917000

0.0000000000

$\odot .0000000000$

$-0.801544000$

3. 125695000

0.000000000

0.529891000

$\odot .0000000000$

$-2.075624000$

0.000000000

$-4.136539000$

3. 878108000

$-0.302888000$

$-3.878108000$

$-0.302888000$

2. 958319000

$-1.559704000$

$-2.958319000$

0.000000000

$-1.559704000$

4.985682000

0.000000000

$-4.143170000$

$\odot .000000000$

3. 499989000

$\odot .000000000$

$\odot .636784000$

0.000000000

$-2.207980000$

2. 958319000

$-1.559704000$

$-2.958319000$

$-1.559704000$

4.985682000

0.000000000

0.000000000

$-4.143170000$

3. 499989000

$\odot .636784000$

0.000000000

$-2.207980000$

$-0.085293000$

1.817861000

$-0.085293000$

1.750589000

0.000000000

$-1.113065000$

1.750589000

0.000000000

$\odot .000000000$

$-1.113065000$

$\odot .000000000$

0.203743000

12. $\left\{\operatorname{Re}[\mathrm{Cor}](\mathrm{NMe})_{2}\right\}^{-;}$ 298.61571916 eV

$\begin{array}{ll}\text { C } & 0.000000000 \\ \text { C } & 0.000000000 \\ \text { C } & 0.000000000 \\ \text { C } & 0.727184000 \\ \text { C } & 1.300833000 \\ \text { C } & 1.847895000 \\ \text { C } & 2.612865000 \\ \text { C } & 2.618255000 \\ \text { C } & 2.858212000\end{array}$
0.000000000
2.993560000
$-2.993560000$
0.000000000
0.0000000000
$\odot .0000000000$
$\odot .000000000$
$\odot .0000000000$
$\odot .0000000000$

$-3.090458000$

$-0.814233000$

$-0.814233000$

3. 007897000

$-2.496685000$

3. 892993000

1.724837000

$-3.112489000$

$-\odot .833321000$ 


\begin{tabular}{|c|c|}
\hline C & $3.0 \odot 57970 \odot \odot$ \\
\hline C & $3.35027400 \odot$ \\
\hline C & $3.55946400 \odot$ \\
\hline C & -0.72718400 \\
\hline C & -1.300833000 \\
\hline C & -1.847895000 \\
\hline $\mathrm{C}$ & -2.61286500 \\
\hline C & -2.61825500 \\
\hline C & -2.85821200 \\
\hline C & -3.00579700 \\
\hline C & -3.350274000 \\
\hline C & $-3.5594640 \odot$ \\
\hline $\mathrm{H}$ & ๑. . 00000000 \\
\hline $\mathrm{H}$ & $\odot .0 \odot \odot \odot \odot \odot \odot \odot \odot$ \\
\hline $\mathrm{H}$ & $\odot . ๑ \odot \odot \odot \odot \odot \odot \odot$ \\
\hline $\mathrm{H}$ & $\odot .8891900 \odot$ \\
\hline $\mathrm{H}$ & ๑. 88919000 \\
\hline $\mathrm{H}$ & 1.81121900 \\
\hline $\mathrm{H}$ & 2.816672000 \\
\hline $\mathrm{H}$ & 4.02501700 \\
\hline $\mathrm{H}$ & 4.43348300 \\
\hline $\mathrm{H}$ & 4.63696900 \\
\hline $\mathrm{H}$ & -0.889190000 \\
\hline $\mathrm{H}$ & -0.88919000 \\
\hline $\mathrm{H}$ & -1.81121900 \\
\hline $\mathrm{H}$ & -2.81667200 \\
\hline $\mathrm{H}$ & -4.02501700 \\
\hline $\mathrm{H}$ & -4.43348300 \\
\hline $\mathrm{H}$ & -4.63696900 \\
\hline $\mathrm{N}$ & 0.00000000 \\
\hline $\mathrm{N}$ & 0.00000000 \\
\hline $\mathrm{N}$ & 1.24909500 \\
\hline $\mathrm{N}$ & 1.53378900 \\
\hline $\mathrm{N}$ & -1.24909500 \\
\hline $\mathrm{N}$ & -1.53378900 \\
\hline $\mathrm{Re}$ & $\odot .000000 \odot \odot$ \\
\hline
\end{tabular}

0.0000000000

3. 110417000

0.000000000

$\odot .501243000$

๑. 0000000000

$-2.104256000$

$\odot .000000000$

3.007897000

0.000000000

$-2.496685000$

$\odot .000000000$

3.892993000

$\odot .0000000000$

1.724837000

0.000000000

0.0000000000

$\odot .0000000000$

$-3.112489000$

$-0.833321000$

3.110417000

0.000000000

0.501243000

$\odot .0000000000$

$-2.104256000$

0.000000000

$-4.178513000$

3. 876960000

$-0.149487000$

$-3.876960000$

$-0.149487000$

3. 089749000

$-1.466934000$

$-3.089749000$

$-1.466934000$

0.000000000

4.976478000

$\odot .000000000$

$-4.178950000$

$\odot .000000000$

3. 481385000

0.000000000

$\odot .600926000$

0.000000000

$-2.229426000$

3. 089749000

$-1.466934000$

$-3.089749000$

$-1.466934000$

4.976478000

0.000000000

0.000000000

$-4.178950000$

0.0000000000

3. 481385000

$\odot .600926000$

0.000000000

$-2.229426000$

0.000000000

$-0.069504000$

$-0.069504000$

$-1.806091000$

1.746690000

$-1.155173000$

$\odot .000000000$

1.746690000

$\odot .00000000 \odot$

$-1.155173000$

$\odot .000000000$

๑. 0000000000

0.176841000

13. $\operatorname{Ir}[\mathrm{Cor}]\left(\mathrm{PMe}_{3}\right) ; C_{\mathrm{s}} ; S=0 ;$ Total bonding energy = $306.08217303 \mathrm{eV}$

$\begin{array}{ll}\text { C } & 0.335913000 \\ C & 0.335913000 \\ C & 0.736254000 \\ \text { C } & 0.736254000 \\ \text { C } & 1.545117000 \\ \text { C } & 1.545117000 \\ C & 2.800505000 \\ \text { C } & 2.800505000 \\ \text { C } & 2.918355000 \\ C & 2.918355000\end{array}$

$-0.498299000$

3.325034000

$-0.498299000$

$-3.325034000$

2.984281000

1.436875000

2.984281000

$-1.436875000$

$-0.429567000$

2.606540000

$-0.429567000$

$-2.606540000$

$-0.385198000$

$\odot .717158000$

$-\odot .385198000$

$-0.717158000$

$-0.636175000$

2. 975881000

$-0.636175000$

$-2.975881000$ 


\begin{tabular}{|c|c|}
\hline C & 3.682600000 \\
\hline C & 3.682600000 \\
\hline C & $-\odot .969119000$ \\
\hline C & $-\odot .969119000$ \\
\hline C & -1.746898000 \\
\hline C & -2.207300000 \\
\hline C & -2.207300000 \\
\hline C & -2.638971000 \\
\hline C & -2.638971000 \\
\hline C & -3.221056000 \\
\hline C & -3.221056000 \\
\hline C & -3.256174000 \\
\hline $\mathrm{H}$ & $\odot .273847000$ \\
\hline $\mathrm{H}$ & $\odot .273847000$ \\
\hline $\mathrm{H}$ & ๑.407540000 \\
\hline $\mathrm{H}$ & $\odot .407540000$ \\
\hline $\mathrm{H}$ & $\odot .608884000$ \\
\hline $\mathrm{H}$ & $\odot .608884000$ \\
\hline $\mathrm{H}$ & 1.800575000 \\
\hline $\mathrm{H}$ & 1.800575000 \\
\hline $\mathrm{H}$ & 3.278702000 \\
\hline $\mathrm{H}$ & 3.278702000 \\
\hline $\mathrm{H}$ & $4.75172000 \odot$ \\
\hline $\mathrm{H}$ & 4.751720000 \\
\hline $\mathrm{H}$ & -1.694539000 \\
\hline $\mathrm{H}$ & -2.287305000 \\
\hline $\mathrm{H}$ & -2.287305000 \\
\hline $\mathrm{H}$ & -2.293931000 \\
\hline $\mathrm{H}$ & -2.293931000 \\
\hline $\mathrm{H}$ & -4.269711000 \\
\hline $\mathrm{H}$ & -4.269711000 \\
\hline $\mathrm{H}$ & -4.329790000 \\
\hline Ir & $\odot .037435000$ \\
\hline $\mathrm{N}$ & 1.540862000 \\
\hline $\mathrm{N}$ & 1.540862000 \\
\hline $\mathrm{N}$ & -1.284716000 \\
\hline $\mathrm{N}$ & -1.284716000 \\
\hline$P$ & $-\odot .05090900 \odot$ \\
\hline
\end{tabular}

$-0.613639000$

$-0.613639000$

$-0.499999000$

- $\odot .499999000$

2.854630000

$-0.732943000$

$-0.732943000$

$-0.576632000$

$-0.576632000$

$-0.780458000$

$-0.780458000$

$-0.633140000$

2. 634015000

2. 634015000

$-0.643003000$

$-0.643003000$

4.068078000

4.068078000

2.744276000

2. 744276000

$-0.819881000$

$-0.819881000$

$-0.776039000$

$-\odot .776039000$

3. 948048000

2. 517872000

2. 517872000

$-\odot .883600000$

$-\odot .88360000 \odot$

$-0.976271000$

$-0.976271000$

$-0.802494000$

$-0.018625000$

$-0.249002000$

$-0.249002000$

$-0.384007000$

$-0.384007000$

2.158097000
1.817036000

$-1.817036000$

2.789686000

$-2.789686000$

๑ . $0 \odot \odot ० ० ० ० \odot \odot$

3.487071000

- 3.487071000

1. 262338000

$-1.262338000$

2. 563267000

$-2.563267000$

0.000000000

2. 361572000

$-2.361572000$

4.400132000

$-4.400132000$

1.353109000

$-1.353109000$

1.465715000

$-1.465715000$

3. 980948000

$-3.980948000$

1. 750487000

$-1.750487000$

๑. . 000000000

$\odot .886302000$

$-0.886302000$

4.556967000

$-4.556967000$

2. 753260000

$-2.753260000$

0.000000000

0.000000000

1. 246459000

$-1.246459000$

1.443403000

$-1.443403000$

0.000000000

14. $\operatorname{Ir}[$ Cor $](\mathrm{CO}) ; C_{\mathrm{s}} ; S=0 ;$ Total bonding energy = $257.47090108 \mathrm{eV}$

$\begin{array}{ll}\text { C } & 0.129664000 \\ \text { C } & 0.129664000 \\ \text { C } & 0.521121000 \\ \text { C } & 0.521121000 \\ \text { C } & 0.937186000 \\ \text { C } & 0.937186000 \\ \text { C } & 1.155194000 \\ \text { C } & 1.155194000\end{array}$

$-0.724445000$

$-0.724445000$

$\odot .515116000$

$\odot .515116000$

1. 644871000

1. 644871000

3. 014498000

3. 014498000
2.788361000

$-2.788361000$

3. 329927000

- 3.329927000

2. 608934000

$-2.608934000$

2.973784000

$-2.973784000$ 


\begin{tabular}{|c|c|c|c|}
\hline C & 1.327569000 & 2.834272000 & $\odot .714125000$ \\
\hline C & 1.327569000 & 2.834272000 & -0.714125000 \\
\hline C & 1.389126000 & 3.739506000 & 1.814203000 \\
\hline C & 1.389126000 & 3.739506000 & -1.814203000 \\
\hline C & 2.632667000 & -0.551625000 & $\odot .000000000$ \\
\hline C & -0.430320000 & -2.296846000 & 1.258393000 \\
\hline C & -0.430320000 & -2.296846000 & -1.258393000 \\
\hline C & -0.441014000 & -1.845730000 & 3.482351000 \\
\hline C & -0.441014000 & -1.845730000 & -3.482351000 \\
\hline C & -0.658297000 & -2.873160000 & 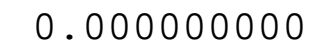 \\
\hline C & -0.780774000 & -2.799845000 & 2.556457000 \\
\hline C & -0.780774000 & -2.799845000 & -2.556457000 \\
\hline $\mathrm{H}$ & $\odot .424115000$ & ๑. 618902000 & 4.407265000 \\
\hline $\mathrm{H}$ & ๑ . 424115000 & ๑. 618902000 & -4.407265000 \\
\hline $\mathrm{H}$ & 1.105170000 & 3.410532000 & 3.980725000 \\
\hline $\mathrm{H}$ & 1.105170000 & 3.410532000 & -3.980725000 \\
\hline $\mathrm{H}$ & 1.553539000 & 4.807973000 & 1.747355000 \\
\hline $\mathrm{H}$ & 1.553539000 & 4.807973000 & -1.747355000 \\
\hline $\mathrm{H}$ & -0.598419000 & -1.891544000 & 4.553402000 \\
\hline $\mathrm{H}$ & $-\odot .598419000$ & -1.891544000 & -4.553402000 \\
\hline H & -1.114134000 & -3.859542000 & 0.000000000 \\
\hline $\mathrm{H}$ & -1.258324000 & -3.753697000 & 2.744868000 \\
\hline $\mathrm{H}$ & -1.258324000 & -3.753697000 & -2.744868000 \\
\hline $\operatorname{Ir}$ & ๑. 951870000 & ० . 068181000 & ๑. . $0000000 \odot \odot$ \\
\hline $\mathrm{N}$ & ๑. 130227000 & -1.045126000 & 1.438516000 \\
\hline $\mathrm{N}$ & 0.130227000 & -1.045126000 & -1.438516000 \\
\hline $\mathrm{N}$ & 1.078384000 & 1.590632000 & 1.242148000 \\
\hline $\mathrm{N}$ & 1.078384000 & 1.590632000 & -1.242148000 \\
\hline 0 & 3.727335000 & -0.959422000 & 0.000000000 \\
\hline
\end{tabular}

\section{5. $\operatorname{Ir}[\mathrm{Cor}](\mathrm{CO})\left(\mathrm{PMe}_{3}\right) ; C_{\mathrm{s}} ; S=0$; Total bonding energy = -} $321.18162849 \mathrm{eV}$

\begin{tabular}{|c|c|}
\hline C & ๑. 631139000 \\
\hline C & $\odot .930145000$ \\
\hline C & 1.10247800 \\
\hline C & 1.10247800 \\
\hline $\mathrm{C}$ & 2.32830200 \\
\hline C & 2.32830200 \\
\hline C & 2.74047900 \\
\hline C & 2.74047900 \\
\hline C & 3.3191720 \\
\hline C & 3.31917200 \\
\hline C & 3.34296900 \\
\hline C & $-0.1722940 \odot$ \\
\hline C & -0.17229400 \\
\hline C & -1.36376700 \\
\hline C & -1.36376700 \\
\hline C & -1.48428400 \\
\hline C & -1.4842840 \\
\hline
\end{tabular}

$$
\begin{array}{r}
2.23478400 \odot \\
-3.20248600 \odot \\
\odot .12445700 \odot \\
\odot .12445700 \odot \\
-\odot .16409700 \odot \\
-\odot .16409700 \odot \\
-\odot .26105100 \odot \\
-\odot .26105100 \odot \\
-\odot .39583200 \odot \\
-\odot .39583200 \odot \\
-\odot .39219900 \odot \\
\odot .4441330 \odot \odot \\
\odot .44413300 \odot \\
\odot .7308050 \odot \odot \\
\odot .73080500 \odot \\
-2.62066800 \odot \\
-2.6206680 \odot \odot
\end{array}
$$
0.000000000
$\odot .0000000 \odot \odot$
2.808437000
$-2.808437000$
3. 515379000
$-3.515379000$
1. 274195000
$-1.274195000$
2. 590997000
$-2.590997000$
0.000000000
3.335109000
$-3.335109000$
2. 617035000
$-2.617035000$
1. 434922000
$-1.434922000$ 


\begin{tabular}{|c|c|}
\hline C & -2.605547000 \\
\hline C & -2.605547000 \\
\hline C & -2.72059900 \\
\hline C & -2.72059900 \\
\hline C & -3.476232000 \\
\hline C & -3.476232000 \\
\hline $\mathrm{H}$ & 0.56624100 \\
\hline $\mathrm{H}$ & 1.54469200 \\
\hline $\mathrm{H}$ & 1.54469200 \\
\hline $\mathrm{H}$ & 2.43073300 \\
\hline $\mathrm{H}$ & 2.43073300 \\
\hline $\mathrm{H}$ & 4.35595100 \\
\hline $\mathrm{H}$ & 4.35595100 \\
\hline $\mathrm{H}$ & 4.404628000 \\
\hline $\mathrm{H}$ & -0.23961300 \\
\hline $\mathrm{H}$ & -0.23961300 \\
\hline $\mathrm{H}$ & $-\odot .93694400$ \\
\hline $\mathrm{H}$ & -0.93694400 \\
\hline $\mathrm{H}$ & -1.68680200 \\
\hline $\mathrm{H}$ & -1.68680200 \\
\hline $\mathrm{H}$ & -2.42930300 \\
\hline $\mathrm{H}$ & -2.42930300 \\
\hline $\mathrm{H}$ & -3.08718800 \\
\hline $\mathrm{H}$ & -3.08718800 \\
\hline $\mathrm{H}$ & -4.53510500 \\
\hline $\mathrm{H}$ & $-4.5351050 \odot$ \\
\hline $\operatorname{Ir}$ & $\odot .1227400 \odot$ \\
\hline $\mathrm{N}$ & 1.41725100 \\
\hline $\mathrm{N}$ & 1.41725100 \\
\hline $\mathrm{N}$ & $-1.3717210 €$ \\
\hline $\mathrm{N}$ & $-1.3717210 €$ \\
\hline 0 & $\odot .9685210 \odot$ \\
\hline$P$ & -0.49464800 \\
\hline
\end{tabular}

1. 001882000

1.001882000

1.019843000

1. 019843000

1.182882000

1.182882000

$-4.234638000$

- 3.036017000

- 3.036017000

$-0.186331000$

$-0.186331000$

$-0.635196000$

$-0.635196000$

$-0.623353000$

0.469236000

0.469236000

$-2.437229000$

$-2.437229000$

$-3.691974000$

$-3.691974000$

$-2.076718000$

$-2.076718000$

1. 093452000

1. 093452000

1. 406942000

1. 406942000

0.450693000

0.031562000

๑. 031562000

0.733973000

0.733973000

3.340524000

$-2.046751000$ $\odot .722631000$

$-0.722631000$

3.000178000

$-3.000178000$

1.842289000

$-1.842289000$

0.000000000

$\odot .885846000$

$-\odot .885846000$

4.594138000

$-4.594138000$

2. 796046000

$-2.796046000$

$\odot .00 \odot \odot \odot \odot \odot \odot \odot$

4.419675000

$-4.419675000$

2.360843000

$-2.360843000$

1.338868000

$-1.338868000$

1.478363000

$-1.478363000$

4. 017162000

$-4.017162000$

1. 796432000

$-1.796432000$

0.000000000

1.467449000

$-1.467449000$

1. 255170000

$-1.255170000$

0.000000000

0.000000000

16. $\operatorname{Re}[\mathrm{Cor}](\mathrm{NMe}) ; C_{\mathrm{s}} ; S=0 ;$ Total bonding energy = 272.26987633 eV

$\begin{array}{lr}\text { C } & 0.430418000 \\ \text { C } & 0.430418000 \\ \text { C } & 1.613801000 \\ \text { C } & 1.613801000 \\ \text { C } & 2.852357000 \\ \text { C } & 2.852357000 \\ \text { C } & 2.986855000 \\ \text { C } & 2.986855000 \\ \text { C } & 3.746029000 \\ \text { C } & 3.746029000 \\ \text { C } & -0.243041000 \\ \text { C } & -0.852718000 \\ \text { C } & -0.852718000\end{array}$

$-\odot .01856200 \odot$
$-\odot .01856200 \odot$
$\odot .07267300 \odot$
$\odot .07267300 \odot$
$\odot .10619500 \odot$
$\odot .1061950 \odot \odot$
$-\odot .0672610 \odot \odot$
$-\odot .06726100 \odot$
$-\odot .0566720 \odot \odot$
$-\odot .05667200 \odot$
$3.89905900 \odot$
$-\odot .0742210 \odot \odot$
$-\odot .07422100 \odot$

3.336529000

$-3.336529000$

2. 594094000

$-2.594094000$

0.708044000

$-0.708044000$

2. 959002000

$-2.959002000$

1.794838000

$-1.794838000$

0.000000000

2.779893000

$-2.779893000$ 


\begin{tabular}{|c|c|}
\hline C & -2.081737000 \\
\hline C & -2.081737000 \\
\hline C & -2.49951800 \\
\hline C & -2.49951800 \\
\hline C & -3.08566100 \\
\hline C & -3.085661000 \\
\hline C & -3.126189000 \\
\hline $\mathrm{H}$ & 0.507714000 \\
\hline $\mathrm{H}$ & 0.50771400 \\
\hline $\mathrm{H}$ & 0.736077000 \\
\hline $\mathrm{H}$ & 3.35680800 \\
\hline $\mathrm{H}$ & 3.35680800 \\
\hline $\mathrm{H}$ & 4.82013600 \\
\hline $\mathrm{H}$ & 4.82013600 \\
\hline $\mathrm{H}$ & -0.796960000 \\
\hline $\mathrm{H}$ & -0.79696000 \\
\hline $\mathrm{H}$ & -2.17237100 \\
\hline $\mathrm{H}$ & -2.17237100 \\
\hline $\mathrm{H}$ & -4.13585000 \\
\hline $\mathrm{H}$ & -4.13585000 \\
\hline $\mathrm{H}$ & -4.19592100 \\
\hline $\mathrm{N}$ & 1.57199700 \\
\hline $\mathrm{N}$ & 1.57199700 \\
\hline$N$ & $-\odot .0822430 \odot$ \\
\hline $\mathrm{N}$ & -1.13879600 \\
\hline $\mathrm{N}$ & -1.13879600 \\
\hline $\mathrm{Re}$ & 0.09 \\
\hline
\end{tabular}

$-0.319821000$

$-0.319821000$

$-0.216269000$

- ๑ . 216269000

- $\odot .408306000$

- 0.408306000

$-0.292929000$

$-0.126510000$

$-\odot .126510000$

4.397013000

$-0.193356000$

$-0.193356000$

$-0.178045000$

$-0.17804500 \odot$

4.223123000

4.223123000

$-0.440094000$

$-0.440094000$

$-0.603966000$

$-0.603966000$

$-0.482077000$

๑. 218515000

๑. 218515000

2. 485901000

๑. . 018580000

๑. 018580000

$\odot .787543000$
3.469251000

$-3.469251000$

1.246845000

$-1.246845000$

2.536586000

$-2.536586000$

0.000000000

4.414727000

$-4.414727000$

๑. . 000000000

3. 969607000

$-3.969607000$

1. 724325000

$-1.724325000$

0.891390000

$-0.891390000$

4.542568000

$-4.542568000$

2.718141000

$-2.718141000$

๑ . $00000000 \odot$

1.223600000

$-1.223600000$

๑ . $0000000 \odot \odot$

1. 414691000

$-1.414691000$

०. $00000000 \odot$

17. $\mathrm{Me}_{3} \mathrm{PO} ; C_{s} ; s=0 ;$ Total bonding energy $=-71.13474396 \mathrm{eV}$

$\begin{array}{lr}\mathrm{C} & 0.996838000 \\ \mathrm{C} & -1.181940000 \\ \mathrm{C} & -1.181940000 \\ \mathrm{H} & 0.418703000 \\ \mathrm{H} & 1.635763000 \\ \mathrm{H} & 1.635763000 \\ \mathrm{H} & -0.585676000 \\ \mathrm{H} & -0.585676000 \\ \mathrm{H} & -1.701601000 \\ \mathrm{H} & -1.701601000 \\ \mathrm{H} & -1.920296000 \\ \mathrm{H} & -1.920296000 \\ \mathrm{O} & 0.654279000 \\ \mathrm{P} & -0.084315000\end{array}$
1.264877000
$\odot .027846000$
0.027846000
2.194424000
1.238321000
1.238321000
$-0.022707000$
$-0.022707000$
0.990599000
$\odot .990599000$
$-\odot .778681000$
$-0.778681000$
$-1.513229000$
$-0.212721000$

0.000000000

1.444482000

$-1.444482000$

0.000000000

$\odot .887097000$

$-0.887097000$

2. 359911000

$-2.359911000$

1.406709000

$-1.406709000$

1.468769000

$-1.468769000$

0.000000000

0.000000000
$\begin{array}{ll}\text { 18. } C 0 ; C_{\text {lin }} S=0 ; \\ \text { C } & 0.0000000000\end{array}$
Total bonding e
energy =
$-14.68008335 \mathrm{eV}$
0
0.0000000000
$\odot .0000000000$
$-1.631366000$
0.000000000
$-2.768853000$ 
19. $\mathrm{PMe}_{3} ; C_{s} ; S=0 ;$ Total bonding energy $=-63.38945444 \mathrm{eV}$

$\begin{array}{rr}\mathrm{C} & 0.979098000 \\ \mathrm{C} & 0.979098000 \\ \mathrm{C} & -1.482165000 \\ \mathrm{H} & 0.469559000 \\ \mathrm{H} & 0.46955900 \odot \\ \mathrm{H} & 1.054302000 \\ \mathrm{H} & 1.054302000 \\ \mathrm{H} & 1.987616000 \\ \mathrm{H} & 1.987616000 \\ \mathrm{H} & -1.291838000 \\ \mathrm{H} & -2.076718000 \\ \mathrm{H} & -2.076718000 \\ \mathrm{P} & 0.067435000\end{array}$
$\odot .154063000$
$\odot .154063000$
0.410540000
$-0.108829000$
$-0.108829000$
1.245640000
1.245640000
$-0.269467000$
$-0.269467000$
1.490231000
0.153935000
0.153935000
$-0.610512000$

1.424873000

$-1.424873000$

0.000000000

2.357579000

$-2.357579000$

1.355135000

$-1.355135000$

1.470474000

$-1.470474000$

0.000000000

0.882830000

$-0.882830000$

0.000000000

20. $\mathrm{MeN}=\mathrm{NMe} ; C_{2 \mathrm{~h}} ; S=0$

Total bonding energy $=-53.36675122 \mathrm{eV}$

$\begin{array}{rr}\mathrm{C} & 1.794402000 \\ \mathrm{C} & -1.794402000 \\ \mathrm{H} & 2.05840700 \odot \\ \mathrm{H} & 2.058407000 \\ \mathrm{H} & 2.34674500 \odot \\ \mathrm{H} & -2.05840700 \odot \\ \mathrm{H} & -2.058407000 \\ \mathrm{H} & -2.34674500 \odot \\ \mathrm{N} & 0.346393000 \\ \mathrm{~N} & -0.346393000\end{array}$

$$
\begin{array}{r}
-0.21073400 \odot \\
0.21073400 \odot \\
0.37426700 \odot \\
0.37426700 \odot \\
-1.15124900 \odot \\
-0.37426700 \odot \\
-0.37426700 \odot \\
1.1512490 \odot \odot \\
-0.51517400 \odot \\
0.51517400 \odot
\end{array}
$$$$
0.0000000000
$$

0.000000000

0.888044000

$-0.888044000$

0.000000000

0.888044000

$-0.888044000$

0.000000000

0.000000000

0.000000000

21. $\mathrm{Me}_{3} \mathrm{P}=\mathrm{NMe} ; C_{1} ; S=0 ;$ Total bonding energy $=-90.40548752 \mathrm{eV}$

$\begin{array}{lr}\mathrm{C} & 1.011408000 \\ \mathrm{C} & 1.897228000 \\ \mathrm{C} & -0.944176000 \\ \mathrm{C} & -1.13360800 \odot \\ \mathrm{H} & 0.35732000 \odot \\ \mathrm{H} & 1.57524400 \odot \\ \mathrm{H} & 1.58414300 \odot \\ \mathrm{H} & 1.71420200 \odot \\ \mathrm{H} & 2.25400100 \odot \\ \mathrm{H} & 2.78243800 \odot \\ \mathrm{H} & -0.28271300 \odot \\ \mathrm{H} & -0.58600700 \odot \\ \mathrm{H} & -1.51788300 \odot \\ \mathrm{H} & -1.63054600 \odot \\ \mathrm{H} & -1.72845500 \odot \\ \mathrm{H} & -1.79213600 \odot\end{array}$

1.352473000

$-2.104860000$

$-0.144618000$

0.001980000

2. 220854000

$-2.363743000$

1. 463448000

1. 318857000

- 3.037627000

$-1.450924000$

- 0.210335000

๑. 062858000

$\odot .785233000$

$-0.995743000$

$\odot .908538000$

$-\odot .869061000$
$-0.048828000$

0.405776000

1.424762000

$-1.472797000$

๑. 078780000

1. 430788000

$-0.974001000$

๑. 789147000

$-0.049234000$

$\odot .506742000$

2. 293910000

$-2.416428000$

1. 491849000

1. 448860000

$-1.332749000$

$-1.510306000$ 


$\begin{array}{lrrr}\text { N } & 0.84443200 \odot & -1.55139300 \odot & -0.42514800 \odot \\ \mathrm{P} & 0.06157700 \odot & -0.230847000 & -0.122847000 \\ & \end{array}$

22. $\operatorname{Ir}[\operatorname{Cor}](C) ; C_{s} ; S=0 ;$ Total bonding energy $=-292.23355312$ eV

\begin{tabular}{|c|c|c|c|}
\hline C & $\odot .133354000$ & $-\odot .709614000$ & $2.783975 c$ \\
\hline C & $\odot .13335400 \odot$ & $-\odot .70961400 \odot$ & -2.78397500 \\
\hline C & $\odot .526678000$ & $\odot .513062000$ & 3.3322970 \\
\hline C & $\odot .526678000$ & $\odot .513062000$ & -3.3322970 \\
\hline C & $\odot .9399860 \odot \odot$ & $1.63616400 \odot$ & 2.6018780 \\
\hline C & $\odot .939986000$ & 1.636164000 & -2.6018780 \\
\hline C & 1.134308000 & 3.005379000 & 2.9586300 \\
\hline C & 1.134308000 & 3.005379000 & $-2.9586300 \mathrm{C}$ \\
\hline C & 1.318669000 & $2.8227200 \odot \odot$ & 0.7100570 \\
\hline C & 1.318669000 & $2.82272000 \odot$ & $-\odot .7100570$ \\
\hline C & 1.357533000 & $3.73044900 \odot$ & 1.7986240 \\
\hline C & 1.357533000 & 3.730449000 & -1.7986240 \\
\hline C & 2.640447000 & $-\odot .593216000$ & $\odot .000000 \odot ८$ \\
\hline C & $-\odot .43107700 \odot$ & -2.271723000 & 1.2485720 \\
\hline C & -0.431077000 & -2.271723000 & -1.2485720 \\
\hline C & $-\odot .4481560 \odot \odot$ & -1.826318000 & 3.4696900 \\
\hline C & $-\odot .448156000$ & -1.826318000 & -3.4696900 \\
\hline C & $-\odot .658997000$ & -2.855284000 & $\odot .0000000 \mathrm{C}$ \\
\hline C & $-\odot .7905120 \odot \odot$ & $-2.77153000 \odot$ & 2.5428130 \\
\hline C & $-\odot .7905120 \odot \odot$ & -2.771530000 & -2.5428130 \\
\hline $\mathrm{H}$ & ๑.428127000 & $\odot .6179650 \odot \odot$ & 4.40441606 \\
\hline $\mathrm{H}$ & $\odot .4281270 \odot \odot$ & $\odot .61796500 \odot$ & -4.4044160 \\
\hline $\mathrm{H}$ & 1.072727000 & 3.401463000 & 3.9595880 \\
\hline $\mathrm{H}$ & $1.07272700 \odot$ & 3.401463000 & -3.9595880 \\
\hline $\mathrm{H}$ & 1.502443000 & 4.796138000 & 1.7273780 \\
\hline $\mathrm{H}$ & 1.502443000 & 4.796138000 & -1.7273780 \\
\hline $\mathrm{H}$ & $-\odot .6077590 \odot \odot$ & -1.871424000 & 4.5354780 \\
\hline $\mathrm{H}$ & $-\odot .6077590 \odot \odot$ & -1.871424000 & -4.5354780 \\
\hline $\mathrm{H}$ & -1.118561000 & -3.834331000 & $\odot .00000 \odot \odot$ \\
\hline $\mathrm{H}$ & -1.272293000 & -3.719277000 & 2.7235400 \\
\hline $\mathrm{H}$ & -1.272293000 & -3.719277000 & -2.7235400 \\
\hline Ir & 1.071490000 & $\odot .026502000$ & $\odot .000000 \odot$ \\
\hline $\mathrm{N}$ & $\odot .139844000$ & -1.032785000 & 1.4345510 \\
\hline $\mathrm{N}$ & $\odot .139844000$ & -1.032785000 & -1.4345510 \\
\hline $\mathrm{N}$ & 1.096891000 & 1.575526000 & 1.2453490 \\
\hline $\mathrm{N}$ & 1.096891000 & 1.575526000 & -1.245349 \\
\hline
\end{tabular}

23. $\operatorname{Ir}[\operatorname{Cor}](C) ; C_{s} ; S=1 ;$ Total bonding energy $=-290.45750677$ eV 


\begin{tabular}{|c|c|}
\hline C & $\odot .139137000$ \\
\hline C & $\odot .13913700 \odot$ \\
\hline C & ๑. $55970500 \odot$ \\
\hline C & ๑. 55970500 \\
\hline C & $\odot .952846000$ \\
\hline C & $\odot .952846000$ \\
\hline C & 1.10697700 \\
\hline C & 1.10697700 \\
\hline C & 1.34962300 \\
\hline C & 1.349623000 \\
\hline C & $1.3550 \odot 90 \odot \odot$ \\
\hline C & 1. 355009000 \\
\hline C & 2.64130800 \\
\hline C & -0.442546000 \\
\hline C & $-\odot .44254600$ \\
\hline C & -0.46078700 \\
\hline C & $-\odot .46 \odot 7870 \odot$ \\
\hline C & $-\odot .6994070 \odot$ \\
\hline C & -0.801376000 \\
\hline C & -0.80137600 \\
\hline $\mathrm{H}$ & $\odot .48397900$ \\
\hline $\mathrm{H}$ & $\odot .48397900$ \\
\hline $\mathrm{H}$ & 1.007570000 \\
\hline $\mathrm{H}$ & $1.0 \odot 7570 \odot \odot$ \\
\hline $\mathrm{H}$ & 1.48223200 \\
\hline $\mathrm{H}$ & 1.48223200 \\
\hline $\mathrm{H}$ & $-\odot .5802500 \odot$ \\
\hline $\mathrm{H}$ & $-\odot .5802500 \odot$ \\
\hline $\mathrm{H}$ & -1.15959100 \\
\hline $\mathrm{H}$ & -1.28357600 \\
\hline $\mathrm{H}$ & -1.28357600 \\
\hline Ir & 1.07889000 \\
\hline $\mathrm{N}$ & ๑. 11981200 \\
\hline $\mathrm{N}$ & ๑. 11981200 \\
\hline $\mathrm{N}$ & 1.14899900 \\
\hline $\mathrm{N}$ & 1.14899900 \\
\hline
\end{tabular}

$-0.703645000$

$-0.703645000$

$\odot .488399000$

๑. 488399000

1.643588000

1. 643588000

3. 019617000

3. 019617000

3. 746464000

3. 746464000

2.836003000

2.836003000

$-0.625041000$

$-1.840666000$

$-1.840666000$

$-2.265004000$

$-2.265004000$

$-2.846692000$

$-2.770050000$

$-2.770050000$

$\odot .589982000$

$\odot .589982000$

3. 413399000

3. 413399000

4.813316000

4.813316000

$-1.892552000$

$-1.892552000$

$-3.825515000$

$-3.718147000$

$-3.718147000$

0.014763000

$-1.018238000$

$-1.018238000$

1.566810000

1.566810000
2.781140000

$-2.781140000$

3. 340116000

- 3.340116000

2.584933000

$-2.584933000$

2. 947098000

$-2.947098000$

1. 786582000

$-1.786582000$

0.705615000

$-0.705615000$

๑. . $00000000 \odot$

3. 473554000

$-3.473554000$

1. 245194000

$-1.245194000$

0.000000000

2.546822000

$-2.546822000$

4.412980000

$-4.412980000$

3. 945319000

- 3.945319000

1. 712531000

$-1.712531000$

4.541698000

$-4.541698000$

๑. . $00 \odot \odot ० ० ० \odot \odot$

2. 726242000

$-2.726242000$

0.000000000

1.427463000

$-1.427463000$

1.254165000

$-1.254165000$

24. $\{\operatorname{Ir}[\operatorname{Cor}](C)\}^{-} ; C_{s} ; S=1 / 2 ;$ Total bonding energy $=$ - $293.50312192 \mathrm{eV}$

$\begin{array}{ll}\text { C } & 0.124716000 \\ \text { C } & 0.124716000 \\ \text { C } & 0.547564000 \\ \text { C } & 0.547564000 \\ \text { C } & 0.935628000 \\ \text { C } & 0.935628000 \\ \text { C } & 1.143638000 \\ \text { C } & 1.143638000 \\ \text { C } & 1.300678000 \\ \text { C } & 1.300678000\end{array}$
$-0.707635000$
$-0.707635000$
0.499344000
0.499344000
1.651812000
1.651812000
3. 000983000
3.000983000
2.849999000
2.849999000

2.792706000

$-2.792706000$

3.350801000

$-3.350801000$

2. 615437000

$-2.615437000$

2. 976905000

$-2.976905000$

$\odot .720802000$

$-0.720802000$ 


\begin{tabular}{|c|c|}
\hline C & 1.363457000 \\
\hline C & 1.363457000 \\
\hline C & 2.604495000 \\
\hline C & -0.43321200 \\
\hline C & -0.433212000 \\
\hline C & -0.461795000 \\
\hline C & $-\odot .4617950 \odot$ \\
\hline C & $-\odot .69060300$ \\
\hline C & $-\odot .78753200$ \\
\hline C & $-\odot .78753200$ \\
\hline $\mathrm{H}$ & $\odot .481227000$ \\
\hline $\mathrm{H}$ & 0.48122700 \\
\hline $\mathrm{H}$ & 1.08898400 \\
\hline $\mathrm{H}$ & 1.08898400 \\
\hline $\mathrm{H}$ & 1.52240000 \\
\hline $\mathrm{H}$ & 1.52240000 \\
\hline $\mathrm{H}$ & $-\odot .5699060 \odot$ \\
\hline $\mathrm{H}$ & -0.56990600 \\
\hline $\mathrm{H}$ & -1.13628000 \\
\hline $\mathrm{H}$ & -1.25673300 \\
\hline $\mathrm{H}$ & -1.25673300 \\
\hline $\operatorname{Ir}$ & 1.03173600 \\
\hline $\mathrm{N}$ & ๑. . 09937600 \\
\hline $\mathrm{N}$ & ๑. . 09937600 \\
\hline $\mathrm{N}$ & 1.06478800 \\
\hline $\mathrm{N}$ & 1 \\
\hline
\end{tabular}

3.741852000

3. 741852000

$-0.569681000$

$-1.840117000$

$-1.840117000$

$-2.273436000$

$-2.273436000$

$-2.852376000$

$-2.782039000$

$-2.782039000$

0.588660000

$\odot .588660000$

3. 395243000

3. 395243000

4.806717000

4.806717000

$-1.898919000$

$-1.898919000$

$-3.839431000$

$-3.737435000$

$-3.737435000$

๑ . 044411000

$-1.015260000$

- 1.015260000

1. 596803000

1. 596803000
1.794735000

$-1.794735000$

0.000000000

3.476402000

- 3.476402000

1. 249907000

$-1.249907000$

0.000000000

2. 540459000

$-2.540459000$

4.427214000

$-4.427214000$

3.979856000

- 3.979856000

1. 726806000

$-1.726806000$

4.545521000

$-4.545521000$

0.000000000

2.722374000

$-2.722374000$

0.000000000

1.437604000

$-1.437604000$

1. 253060000

$-1.253060000$

25. $\operatorname{Ir}[\mathrm{Cor}](\mathrm{NMe})_{2} ; C_{2 \mathrm{v}} ;$ $345.12164958 \mathrm{eV}$

\begin{tabular}{|c|c|}
\hline C & ๑. . 000000000 \\
\hline C & ๑. .००००००००० \\
\hline C & ๑. . 000000000 \\
\hline C & ๑. 71979600 \\
\hline C & 1.27256200 \\
\hline C & 1.843298000 \\
\hline C & 2.589826000 \\
\hline C & 2.61189600 \\
\hline C & 2.801453000 \\
\hline C & 2.99589700 \\
\hline $\mathrm{C}$ & 3.32367500 \\
\hline C & 3.51012100 \\
\hline C & -0.71979600 \\
\hline C & -1.27256200 \\
\hline C & -1.84329800 \\
\hline C & -2.58982600 \\
\hline C & -2.61189600 \\
\hline C & -2.80145300 \\
\hline C & -2.99589700 \\
\hline C & -3.32367500 \\
\hline
\end{tabular}
0.000000000
2.781972000
$-2.781972000$
๑. 000000000
๑ . $000000 \odot \odot \odot$
๑. 000000000
$\odot .000000000$
๑. $.0000 \odot \odot ० ० \odot$
0.000000000
०. 000000000
$\odot .000000000$
$\odot .00000000 \odot$
0.000000000
$\odot .000000000$
๑. 000000000
$\odot .00000000 \odot$
๑. 000000000
๑. 000000000
$\odot .000000000$
๑. $00000000 \odot$

$-3.086513000$

$-\odot .939358000$

$-0.939358000$

3. 026962000

$-2.470494000$

3. 906402000

$-3.062920000$

1. 746450000

$-0.788802000$

3.130927000

$\odot .525377000$

$-2.046716000$

3. 026962000

$-2.470494000$

3. 906402000

- 3.062920000

1.746450000

$-0.788802000$

3.130927000

$\odot .525377000$ 


\begin{tabular}{|c|c|}
\hline C & -3.510121000 \\
\hline $\mathrm{H}$ & 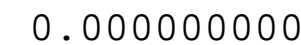 \\
\hline $\mathrm{H}$ & ๑. .००००००८ \\
\hline $\mathrm{H}$ & $\odot .0 \odot \odot \odot \odot \odot \odot \odot$ \\
\hline $\mathrm{H}$ & 0.88786300 \\
\hline $\mathrm{H}$ & ๑. 88786300 \\
\hline $\mathrm{H}$ & 1.80418500 \\
\hline $\mathrm{H}$ & 2.79410600 \\
\hline $\mathrm{H}$ & 4.008790000 \\
\hline $\mathrm{H}$ & 4.40381100 \\
\hline $\mathrm{H}$ & 4.58403200 \\
\hline $\mathrm{H}$ & -0.88786300 \\
\hline $\mathrm{H}$ & -0.887863000 \\
\hline $\mathrm{H}$ & -1.80418500 \\
\hline $\mathrm{H}$ & -2.79410600 \\
\hline $\mathrm{H}$ & $-4.0 \odot 8790 \odot \odot$ \\
\hline $\mathrm{H}$ & -4.40381100 \\
\hline $\mathrm{H}$ & -4.58403200 \\
\hline $\operatorname{Ir}$ & 0.00000000 \\
\hline $\mathrm{N}$ & ०. . 00000000 \\
\hline $\mathrm{N}$ & ๑. . 00000000 \\
\hline $\mathrm{N}$ & 1.25180600 \\
\hline $\mathrm{N}$ & 1.47103700 \\
\hline $\mathrm{N}$ & -1.25180600 \\
\hline $\mathrm{N}$ & -1.47103706 \\
\hline
\end{tabular}

$\odot . ๑ \odot \odot \odot \odot \odot \odot \odot ९$

$\odot .000000000$

3. 772237000

$-3.772237000$

2.704768000

$-2.704768000$

0.000000000

๑. . $0000000 \odot \odot$

๑ . $.00000 \odot ० \odot$

๑. 000000000

๑. $00000000 \odot$

2. 704768000

$-2.704768000$

๑. . $00 \odot \odot ० ० ० \odot \odot$

0.000000000

$\odot .00000000 \odot$

$\odot .00000000 \odot$

○. $00000000 \odot$

๑ . 000000000

1. 798704000

$-1.798704000$

๑ . $00000000 \odot$

๑. $0000000 \odot \odot$

๑ . $00 \odot \odot \odot ० ० \odot \odot$

๑. 000000000
$-2.046716000$

$-4.168650000$

$-0.471811000$

$-0.471811000$

$-1.582361000$

$-1.582361000$

4.983713000

$-4.121922000$

3. 500990000

$\odot .595721000$

$-2.149369000$

$-1.582361000$

$-1.582361000$

4.983713000

$-4.121922000$

3. 500990000

$\odot .595721000$

$-2.149369000$

๑. 265142000

๑ . 069118000

๑. 069118000

1.767449000

$-1.122575000$

1.767449000

$-1.122575000$

26. $\operatorname{Ir}[\mathrm{Cor}](\mathrm{NMe})_{2} ; C_{2 v} ;$ 343.71958688 eV

\begin{tabular}{|c|c|}
\hline C & ๑. . 000000000 \\
\hline C & 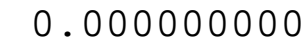 \\
\hline C & ๑. . 000000000 \\
\hline C & 0.73728200 \\
\hline C & 1.279442000 \\
\hline C & 1.852398000 \\
\hline C & 2.59051500 \\
\hline C & 2.61757300 \\
\hline C & 2.790008000 \\
\hline C & 3.00704300 \\
\hline C & 3.31637300 \\
\hline C & 3.51098300 \\
\hline C & $-0.7372820 \odot$ \\
\hline C & -1.27944200 \\
\hline C & -1.85239800 \\
\hline C & -2.59051500 \\
\hline C & -2.61757300 \\
\hline C & -2.79000800 \\
\hline C & $-3.0070430 \odot$ \\
\hline C & -3.31637300 \\
\hline 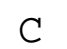 & -3 \\
\hline
\end{tabular}
0.000000000
3. 236879000
$-3.236879000$
0.000000000
๑ . $0000000 \odot \odot$
๑ . $000000 \odot \odot \odot$
๑. 000000000
$\odot .0000000 \odot \odot$
๑. $.0000 \odot \odot ० ० \odot$
0.000000000
$\odot .000000000$
$\odot .000000000$
$\odot .0000000 \odot \odot$
0.000000000
$\odot .000000000$
0.000000000
$\odot .000000 \odot \odot \odot$
๑ . 000000000
๑. $0000000 \odot \odot$
๑. . $00000000 \odot$
๑. $00000000 \odot$

$-3.327887000$

$-0.016520000$

$-0.016520000$

2. 748022000

$-2.720671000$

3. 651046000

$-3.322793000$

1.495153000

$-1.054675000$

2. 885567000

0.275720000

$-2.305750000$

2. 748022000

$-2.720671000$

3.651046000

$-3.322793000$

1.495153000

$-1.054675000$

2.885567000

๑. 275720000

$-2.305750000$ 


\begin{tabular}{|c|c|}
\hline $\mathrm{H}$ & 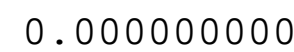 \\
\hline $\mathrm{H}$ & 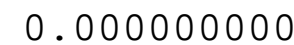 \\
\hline $\mathrm{H}$ & ๑ . ๑००००००८ \\
\hline $\mathrm{H}$ & ๑. .88967900 \\
\hline $\mathrm{H}$ & ๑. 88967900 \\
\hline $\mathrm{H}$ & 1.800042000 \\
\hline $\mathrm{H}$ & 2.79294100 \\
\hline $\mathrm{H}$ & 4.01896800 \\
\hline $\mathrm{H}$ & 4.397789000 \\
\hline $\mathrm{H}$ & 4.58495100 \\
\hline $\mathrm{H}$ & -0.88967900 \\
\hline $\mathrm{H}$ & $-\odot .8896790 \odot$ \\
\hline $\mathrm{H}$ & -1.80004200 \\
\hline $\mathrm{H}$ & -2.79294100 \\
\hline $\mathrm{H}$ & - 4.01896800 \\
\hline $\mathrm{H}$ & -4.39778900 \\
\hline $\mathrm{H}$ & -4.58495100 \\
\hline Ir & $\odot .0000 \odot \odot \odot \odot$ \\
\hline $\mathrm{N}$ & 0.00000000 \\
\hline $\mathrm{N}$ & 0.00000000 \\
\hline $\mathrm{N}$ & 1.25896600 \\
\hline$N$ & 1.47074200 \\
\hline$N$ & -1.25896600 \\
\hline $\mathrm{N}$ & -1.47074200 \\
\hline
\end{tabular}

$\odot .00000000 \odot$

3. 617548000

$-3.617548000$

3. 672529000

$-3.672529000$

๑. . 000000000

๑. 000000000

๑. . $0000000 \odot \odot$

๑ . $.00000 \odot ० \odot$

๑. 000000000

3. 672529000

$-3.672529000$

$\odot .0000000 \odot \odot$

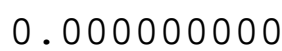

$\odot .000000000$

$\odot .00000000 \odot$

$\odot .00000000 \odot$

$\odot .000000000$

1.843492000

$-1.843492000$

๑ . 000000000

๑. . $0000000 \odot \odot$

๑. $0000000 \odot \odot$

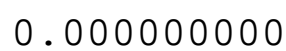

$-4.411108000$

1.018516000

1. 018516000

$-0.503182000$

$-0.503182000$

4.727506000

$-4.381939000$

3. 257805000

๑. 338635000

$-2.404626000$

$-0.503182000$

$-0.503182000$

4.727506000

$-4.381939000$

3. 257805000

๑. 338635000

$-2.404626000$

- $\odot .015613000$

$-0.023253000$

$-0.023253000$

1. 511068000

$-1.375735000$

1. 511068000

$-1.375735000$

27. $\left\{\operatorname{Ir}[\text { Cor }](\text { NMe })_{2}\right\}^{-;}$ $346.84301678 \mathrm{eV}$

\begin{tabular}{|c|c|}
\hline C & ๑. . 000000000 \\
\hline C & ๑. . 000000000 \\
\hline C & $\odot . \odot \odot \odot \odot \odot \odot \odot \odot$ \\
\hline $\mathrm{C}$ & ๑. 72939400๑ \\
\hline $\mathrm{C}$ & 1. 266735000 \\
\hline C & 1.845846000 \\
\hline C & 2.587355000 \\
\hline $\mathrm{C}$ & 2.610648000 \\
\hline C & 2.78764900 \\
\hline C & 3.003939000 \\
\hline C & 3.31614000 \\
\hline $\mathrm{C}$ & 3.50396900 \\
\hline C & $-\odot .7293940 \odot$ \\
\hline C & -1.26673500 \\
\hline C & -1.84584600 \\
\hline C & -2.58735500 \\
\hline C & -2.61064800 \\
\hline C & -2.78764900 \\
\hline C & $-3.0 \odot 3939 \odot \odot$ \\
\hline C & -3.31614000 \\
\hline C & -3.50396900 \\
\hline $\mathrm{H}$ & 0.0 \\
\hline
\end{tabular}

$\odot .00000000 \odot$

2. 869441000

$-2.869441000$

๑. 000000000

๑. 000000000

๑. 000000000

๑ . $0000 \odot ० ० ० \odot$

๑. 000000000

0.000000000

๑. $.0000 \odot \odot ० ० \odot$

$\odot .00000 \odot \odot \odot \odot$

०. 000000000

$\odot .00000000 \odot$

$\odot .0 \odot \odot \odot ० ० \odot \odot \odot$

$\odot .00000000 \odot$

$\odot .000000000$

0.000000000

๑. . $0000000 \odot \odot$

०. $00000000 \odot$

๑. $0000000 \odot \odot$

๑. $00000000 \odot$

$\odot .0 \odot \odot \odot ० ० \odot \odot \odot$
$-3.594955000$

0.890178000

๑. 890178000

2. 513797000

$-2.973202000$

3. 404925000

$-3.567793000$

1. 246335000

$-1.293208000$

2. 629128000

0.010982000

$-2.550940000$

2. 513797000

$-2.973202000$

3. 404925000

$-3.567793000$

1. 246335000

$-1.293208000$

2. 629128000

0.010982000

$-2.550940000$

$-4.678036000$ 


\begin{tabular}{|c|c|}
\hline $\mathrm{H}$ & ๑. .००००००००८ \\
\hline $\mathrm{H}$ & 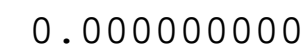 \\
\hline $\mathrm{H}$ & ๑.89013000 \\
\hline $\mathrm{H}$ & ๑. 89013000९ \\
\hline $\mathrm{H}$ & 1.80094700 \\
\hline $\mathrm{H}$ & 2.79252800 \\
\hline $\mathrm{H}$ & 4.01802600 \\
\hline $\mathrm{H}$ & 4.39816100 \\
\hline $\mathrm{H}$ & 4.579372000 \\
\hline $\mathrm{H}$ & $-\odot .89013000$ \\
\hline $\mathrm{H}$ & -0.89013000 \\
\hline $\mathrm{H}$ & -1.80094700 \\
\hline $\mathrm{H}$ & -2.79252800 \\
\hline $\mathrm{H}$ & -4.01802600 \\
\hline $\mathrm{H}$ & -4.39816100 \\
\hline $\mathrm{H}$ & $-4.5793720 \odot$ \\
\hline $\operatorname{Ir}$ & 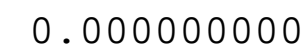 \\
\hline $\mathrm{N}$ & ๑. . \\
\hline $\mathrm{N}$ & 0.00000000 \\
\hline $\mathrm{N}$ & 1.25996900 \\
\hline $\mathrm{N}$ & 1.45347600 \\
\hline $\mathrm{N}$ & -1.25996900 \\
\hline $\mathrm{N}$ & -1.4 \\
\hline
\end{tabular}

2.429582000

$-2.429582000$

3. 521929000

- 3.521929000

๑ . $0000000 \odot \odot$

๑ . 000000000

○. 000000000

$\odot .000000000$

๑ . $0000000 \odot \odot$

3. 521929000

$-3.521929000$

0.000000000

๑. . 000000000

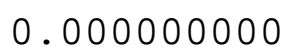

$\odot .00000000 \odot$

$\odot .00000000 \odot$

$\odot .000000000$

1.880904000

$-1.880904000$

๑ . $00000 \odot \odot \odot \odot$

๑. . 000000000

๑. 000000000

๑. $000000 \odot \odot \odot$
1.901531000

1.901531000

0.839453000

๑. 839453000

4.482952000

$-4.627711000$

2. 999124000

๑. 072533000

$-2.649279000$

$\odot .839453000$

$\odot .839453000$

4.482952000

$-4.627711000$

2. 999124000

$\odot .072533000$

$-2.649279000$

$-0.275361000$

$-0.110094000$

$-0.110094000$

1.263695000

$-1.628274000$

1.263695000

$-1.628274000$

28. $\operatorname{Re}[$ Cor $](C) ; C_{s} ; S=0 ;$ Total bonding energy $=-293.35782102$ eV

\begin{tabular}{|c|c|}
\hline C & ๑. 328661000 \\
\hline C & ๑. 328661000 \\
\hline C & ๑.897583000 \\
\hline C & ๑. 89758300 \\
\hline C & 1.070754000 \\
\hline C & 1.070754000 \\
\hline C & 1.470325000 \\
\hline C & 1.470325000 \\
\hline C & 1.56210300 \\
\hline C & 1.562103000 \\
\hline C & 2.76484900 \\
\hline C & $-0.0226410 \odot$ \\
\hline C & $-0.0226410 \odot$ \\
\hline C & $-\odot .24194600$ \\
\hline C & $-0.2419460 \odot$ \\
\hline C & -0.39014500 \\
\hline C & -0.62416600 \\
\hline C & -0.62416600 \\
\hline C & -0.74173600 \\
\hline C & -0.74173600 \\
\hline $\mathrm{H}$ & 0.10474600 \\
\hline $\mathrm{H}$ & 0.10474600 \\
\hline $\mathrm{H}$ & 0.85294000 \\
\hline
\end{tabular}

๑. 605038000

0.605038000

1.655756000

1.655756000

3. 042286000

3.042286000

3.704655000

3. 704655000

2. 732441000

2.732441000

$-0.846448000$

$-0.653562000$

$-0.653562000$

$-2.322371000$

$-2.322371000$

$-2.899663000$

$-1.776572000$

$-1.776572000$

$-2.796242000$

$-2.796242000$

$\odot .783976000$

$\odot .783976000$

3. 485240000
3.297784000

- 3.297784000

2. 571333000

$-2.571333000$

2. 900181000

$-2.900181000$

1.757959000

$-1.757959000$

$\odot .719258000$

$-0.719258000$

0.000000000

2.783297000

$-2.783297000$

1. 276869000

$-1.276869000$

0.000000000

3.443532000

- 3.443532000

2. 536162000

$-2.536162000$

4.341469000

$-4.341469000$

3. 859055000 


$\begin{array}{lr}\mathrm{H} & 0.85294000 \odot \\ \mathrm{H} & 1.63280600 \odot \\ \mathrm{H} & 1.63280600 \odot \\ \mathrm{H} & -\odot .81214800 \odot \\ \mathrm{H} & -0.93573100 \odot \\ \mathrm{H} & -\odot .93573100 \odot \\ \mathrm{H} & -1.16822800 \odot \\ \mathrm{H} & -1.16822800 \odot \\ \mathrm{N} & \odot .21503900 \odot \\ \mathrm{N} & \odot .21503900 \odot \\ \mathrm{N} & 1.27239100 \odot \\ \mathrm{N} & 1.27239100 \odot \\ \mathrm{Re} & 1.22754800 \odot\end{array}$

3.485240000

4.764568000

4.764568000

- 3.897506000

$-1.788743000$

$-1.788743000$

$-3.772142000$

$-3.772142000$

$-1.034042000$

$-1.034042000$

1. 517714000

1.517714000

$-0.116318000$
- 3.859055000

1.650436000

$-1.650436000$

०. . 000000000

4.476017000

$-4.476017000$

2.705837000

$-2.705837000$

1. 481757000

$-1.481757000$

1. 256763000

$-1.256763000$

๑. . $00000000 \odot$

29. $\operatorname{Re}[\operatorname{Cor}](C) ; C_{s} ; S=1 ;$ Total bonding energy $=-293.52018590$ eV

\begin{tabular}{|c|c|}
\hline & $\odot .201078000$ \\
\hline & $\odot .20107800 \odot$ \\
\hline & $\odot .56326500 \odot$ \\
\hline & $\odot .56326500 \odot$ \\
\hline & 1.017636000 \\
\hline & 1.017636000 \\
\hline & 1.034944000 \\
\hline & 1.034944000 \\
\hline & 1.294863000 \\
\hline & 1.294863000 \\
\hline & $1.45224400 \odot$ \\
\hline & $1.45224400 \odot$ \\
\hline & 2.925536000 \\
\hline & $-\odot .3745080 \odot \odot$ \\
\hline & -0.374508000 \\
\hline & -0.502942000 \\
\hline & $-0.50294200 \odot$ \\
\hline & -0.634255000 \\
\hline & $-\odot .85573600 \odot$ \\
\hline & $-\odot .85573600 \odot$ \\
\hline & $\odot .364177000$ \\
\hline & $\odot .364177000$ \\
\hline & $\odot .83140300 \odot$ \\
\hline & $\odot .83140300 \odot$ \\
\hline & 1.333468000 \\
\hline & 1.333468000 \\
\hline & $-\odot .7356390 \odot \odot$ \\
\hline & -0.735639000 \\
\hline & -1.188642000 \\
\hline & -1.421969000 \\
\hline & -1.421969000 \\
\hline & $\odot .302085000$ \\
\hline & $\odot .302085000$ \\
\hline
\end{tabular}

$-0.734469000$

$-0.734469000$

0.499459000

0.499459000

1.617688000

1.617688000

3. 028282000

3.028282000

3.735205000

3.735205000

2.783618000

2.783618000

$-0.743427000$

$-2.271734000$

$-2.271734000$

$-1.803995000$

$-1.803995000$

$-2.840051000$

$-2.729459000$

$-2.729459000$

0.658494000

0.658494000

3.435689000

3.435689000

4.807230000

4.807230000

$-1.811251000$

$-1.811251000$

$-3.768575000$

$-3.633312000$

$-3.633312000$

$-1.107725000$

$-1.107725000$
2.797038000

$-2.797038000$

3. 319047000

$-3.319047000$

2.568005000

$-2.568005000$

2. 905156000

$-2.905156000$

1.759714000

$-1.759714000$

0.699963000

$-0.699963000$

0.000000000

1.256781000

$-1.256781000$

3.477838000

$-3.477838000$

0.0000000000

2. 550287000

$-2.550287000$

4.370340000

$-4.370340000$

3.882705000

$-3.882705000$

1.656288000

$-1.656288000$

4.530774000

$-4.530774000$

0.0000000000

2.710487000

$-2.710487000$

1. 461929000

$-1.461929000$ 


$\begin{array}{lrrr}\mathrm{N} & 1.348878000 & 1.516789000 & 1.268669000 \\ \mathrm{~N} & 1.348878000 & 1.516789000 & -1.268669000 \\ \mathrm{Re} & 1.326775000 & -0.092897000 & 0.000000000\end{array}$

30. $\{\operatorname{Re}[\operatorname{Cor}](C)\}^{-} ; C_{s} ; S=1 / 2 ;$ Total bonding energy = $296.62542075 \mathrm{eV}$

\begin{tabular}{|c|c|c|c|}
\hline C & ๑. 196318000 & $-0.73641000 \odot$ & 2.797861000 \\
\hline C & ๑ . 196318000 & -0.736410000 & -2.797861000 \\
\hline C & 0.570480000 & $\odot .505561000$ & 3.326551000 \\
\hline C & $\odot .570480000$ & ๑.50556100९ & -3.326551000 \\
\hline C & 1.030757000 & 1.621589000 & 2.597306000 \\
\hline C & 1.030757000 & 1.621589000 & -2.597306000 \\
\hline C & 1.057591000 & 3.014335000 & 2.935902000 \\
\hline C & 1.057591000 & 3.014335000 & -2.935902000 \\
\hline C & 1.288977000 & 3.729020000 & 1.770555000 \\
\hline C & 1.288977000 & 3.729020000 & -1.770555000 \\
\hline C & 1.424486000 & 2.784123000 & $\odot .715650000$ \\
\hline C & 1.424486000 & 2.784123000 & -0.715650000 \\
\hline C & 2.935632000 & $-\odot .7209390 \odot \odot$ & ๑. ๑००००००००९ \\
\hline C & -0.362904000 & -2.285919000 & 1. 261984000 \\
\hline C & $-\odot .36290400 \odot$ & $-2.2859190 \odot \odot$ & -1.261984000 \\
\hline C & $-\odot .51472400 \odot$ & $-1.79183000 \odot$ & 3.471571000 \\
\hline C & -0.514724000 & $-1.79183000 \odot$ & -3.471571000 \\
\hline C & -0.616398000 & -2.850070000 & 0.000000000 \\
\hline C & -0.854402000 & -2.735011000 & 2.540468000 \\
\hline C & -0.854402000 & -2.735011000 & -2.540468000 \\
\hline $\mathrm{H}$ & ๑. 364175000 & $\odot .656857000$ & 4.379230000 \\
\hline $\mathrm{H}$ & $\odot .364175000$ & ๑. $65685700 \odot$ & $-4.37923000 \odot$ \\
\hline $\mathrm{H}$ & ๑. 864262000 & 3.424966000 & 3.915388000 \\
\hline $\mathrm{H}$ & ๑. 864262000 & 3.424966000 & -3.915388000 \\
\hline $\mathrm{H}$ & 1.318216000 & 4.802767000 & 1.668931000 \\
\hline $\mathrm{H}$ & 1.318216000 & 4.802767000 & -1.668931000 \\
\hline $\mathrm{H}$ & -0.764557000 & -1.792698000 & 4.521918000 \\
\hline $\mathrm{H}$ & -0.764557000 & -1.792698000 & -4.521918000 \\
\hline $\mathrm{H}$ & -1.169143000 & -3.781298000 & 0.000000000 \\
\hline $\mathrm{H}$ & -1.427864000 & -3.635394000 & 2.700985000 \\
\hline $\mathrm{H}$ & -1.427864000 & $-3.6353940 \odot \odot$ & -2.700985000 \\
\hline $\mathrm{N}$ & $\odot .307999000$ & -1.110082000 & 1.472738000 \\
\hline $\mathrm{N}$ & ๑ . 307999000 & -1.110082000 & -1.472738000 \\
\hline $\mathrm{N}$ & 1.323378000 & 1.536861000 & 1.266855000 \\
\hline $\mathrm{N}$ & 1.323378000 & 1.536861000 & -1.266855000 \\
\hline $\mathrm{Re}$ & 1.341441000 & $-\odot .08909800 \odot$ & $\odot .000 \odot \odot \odot \odot ० \odot$ \\
\hline
\end{tabular}

31. $\operatorname{Re}[\mathrm{Cor}](\mathrm{NMe})_{2} ; C_{2 \mathrm{v}} ; S=0 ;$ Total bonding energy $=-$ $346.20155412 \mathrm{eV}$
C $\quad 0.000000000$
0.000000000
$-2.917868000$
C $\quad 0.0000000000$
1.743534000
$-0.335068000$ 


\begin{tabular}{|c|c|}
\hline C & 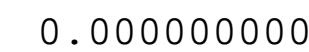 \\
\hline C & 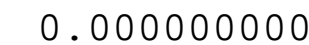 \\
\hline C & 0.00000000 \\
\hline C & $\odot .72390000 \odot$ \\
\hline C & 1.318545000 \\
\hline C & 1.84198000 \\
\hline C & 2.62152500 \\
\hline C & 2.63331200 \\
\hline C & $2.91678000 \odot$ \\
\hline C & 2.997465000 \\
\hline C & 3.38417400 \\
\hline C & 3.58844200 \\
\hline C & -0.72390000 \\
\hline C & -1.318545000 \\
\hline C & -1.84198000 \\
\hline C & -2.62152500 \\
\hline C & -2.63331200 \\
\hline C & $-2.91678000 \odot$ \\
\hline C & -2.997465000 \\
\hline C & -3.38417400 \\
\hline C & -3.58844200 \\
\hline $\mathrm{H}$ & 0.000000000 \\
\hline $\mathrm{H}$ & $\odot .0 \odot \odot ० ० \odot ० \odot$ \\
\hline $\mathrm{H}$ & ๑. . 00000000 \\
\hline $\mathrm{H}$ & $\odot .87845800$ \\
\hline $\mathrm{H}$ & $\odot .87845800$ \\
\hline $\mathrm{H}$ & 1.801225000 \\
\hline $\mathrm{H}$ & 2.81664600 \\
\hline $\mathrm{H}$ & 4.00930800 \\
\hline $\mathrm{H}$ & 4. 45961000 \\
\hline $\mathrm{H}$ & 4.657446000 \\
\hline $\mathrm{H}$ & $-0.8784580 \odot$ \\
\hline $\mathrm{H}$ & -0.87845800 \\
\hline $\mathrm{H}$ & -1.80122500 \\
\hline $\mathrm{H}$ & -2.81664600 \\
\hline $\mathrm{H}$ & -4.00930800 \\
\hline $\mathrm{H}$ & -4.45961000 \\
\hline $\mathrm{H}$ & -4.65744600 \\
\hline$N$ & 1.24959100 \\
\hline $\mathrm{N}$ & 1.59431600 \\
\hline $\mathrm{N}$ & -1.24959100 \\
\hline $\mathrm{N}$ & -1.59431600 \\
\hline $\mathrm{Re}$ & $\odot .00000006$ \\
\hline
\end{tabular}

2.826838000

$-1.743534000$

$-2.826838000$

๑. . 000000000

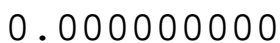

๑. . 000000000

๑. $00000000 \odot$

$\odot .000000000$

๑ . $.0000000 \odot \odot$

๑. . 000000000

๑. $0000000 \odot \odot$

$\odot .00000000 \odot$

๑. . 000000000

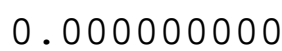

0.000000000

०. $00000000 \odot$

$\odot .00000000 \odot$

0.000000000

๑. . 000000000

๑ . $00 \odot ० ० ० ० ० \odot$

๑ . $00000000 \%$

○. 000000000

3.806839000

- 3.806839000

2. 736922000

$-2.736922000$

๑. $00000000 \odot$

๑. . $00 \odot ० ० ० \odot \odot \odot$

๑. . $000 \odot ० ० ० ० \odot$

๑. . $000 \odot \odot ० ० ० \odot$

○. $00000000 \odot$

2. 736922000

$-2.736922000$

๑. 000000000

๑. . $0000000 \odot \odot$

$\odot .000000 \odot \odot \odot$

๑. . 000000000

๑. . 000000000

๑ . $0000 \odot \odot ० ० \odot$

๑. . 000000000

๑. $.0000000 \odot \odot$

$\odot .00 \odot \odot \odot \odot ० \odot \odot$

๑. . $00000000 \odot$
$-1.280716000$

$-0.335068000$

$-1.280716000$

3. 122192000

$-2.344367000$

4.004966000

1.845367000

$-2.970165000$

$-\odot .693293000$

3. 231589000

$\odot .643068000$

$-1.981811000$

3. 122192000

$-2.344367000$

4.004966000

1.845367000

$-2.970165000$

$-0.693293000$

3. 231589000

0.643068000

$-1.981811000$

$-4.001592000$

$-0.796566000$

$-\odot .796566000$

$-1.933529000$

$-1.933529000$

5.082351000

$-4.033553000$

3. 605490000

๑. 768828000

$-2.127313000$

$-1.933529000$

$-1.933529000$

5.082351000

$-4.033553000$

3. 605490000

$\odot .768828000$

$-2.127313000$

1.859648000

$-1.004684000$

1.859648000

$-1.004684000$

0.300464000

32. $\operatorname{Re}[\mathrm{Cor}](\mathrm{NMe})_{2} ; C_{2 v} ; S=1 ;$ Total bonding energy $=--$ $345.77665802 \mathrm{eV}$
C
0.000000000
0.000000000
$-2.976886000$
1.821622000
$-0.264546000$
C
$\odot .000000000$
3. 017209000
$-1.094625000$ 


\begin{tabular}{|c|c|}
\hline C & 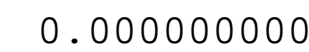 \\
\hline C & 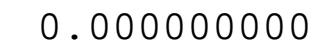 \\
\hline C & 0.73541200 \\
\hline C & 1.31987500 \\
\hline $\mathrm{C}$ & 1.858893000 \\
\hline $\mathrm{C}$ & 2.621906000 \\
\hline $\mathrm{C}$ & 2.63024000 \\
\hline C & 2.88060900 \\
\hline C & 3.00870900 \\
\hline $\mathrm{C}$ & 3.35697500 \\
\hline $\mathrm{C}$ & 3.57696500 \\
\hline $\mathrm{C}$ & -0.73541200 \\
\hline C & -1.31987500 \\
\hline C & -1.85889300 \\
\hline C & -2.621906000 \\
\hline C & -2.630240000 \\
\hline C & -2.88060900 \\
\hline C & -3.008709000 \\
\hline C & -3.35697500 \\
\hline C & -3.57696500 \\
\hline $\mathrm{H}$ & ๑. . 00000000 \\
\hline $\mathrm{H}$ & 0.00000000 \\
\hline $\mathrm{H}$ & 0.000000000 \\
\hline $\mathrm{H}$ & $\odot .88753200$ \\
\hline $\mathrm{H}$ & 0.88753200 \\
\hline $\mathrm{H}$ & 1.81439600 \\
\hline $\mathrm{H}$ & 2.81930900 \\
\hline $\mathrm{H}$ & 4.021138000 \\
\hline $\mathrm{H}$ & 4.43502700 \\
\hline $\mathrm{H}$ & 4.64736700 \\
\hline $\mathrm{H}$ & -0.88753200 \\
\hline $\mathrm{H}$ & -0.88753200 \\
\hline $\mathrm{H}$ & -1.81439600 \\
\hline $\mathrm{H}$ & -2.81930900 \\
\hline $\mathrm{H}$ & -4.02113800 \\
\hline $\mathrm{H}$ & -4.43502700 \\
\hline $\mathrm{H}$ & -4.64736700 \\
\hline $\mathrm{N}$ & 1.24631300 \\
\hline $\mathrm{N}$ & 1.57074100 \\
\hline $\mathrm{N}$ & -1.24631300 \\
\hline $\mathrm{N}$ & -1.57074100 \\
\hline $\mathrm{Re}$ & $\odot$. \\
\hline
\end{tabular}

$-1.821622000$

$-3.017209000$

0.000000000

$\odot .000000000$

$\odot .000000000$

0.000000000

$\odot .00000000 \odot$

$\odot .000000000$

$\odot .00000000 \odot$

$\odot .00000000 \odot$

๑ . $0000000 \odot \odot$

๑. 000000000

$\odot .00000 \odot \odot \odot \odot$

๑. . $00 \odot ० ० ० ० \odot \odot$

๑. 000000000

๑. $00000000 \odot$

0.000000000

○. 000000000

๑ . 000000000

$\odot .000000000$

๑ . 000000000

3. 915609000

$-3.915609000$

3. 065437000

$-3.065437000$

๑ . $00000000 \odot$

๑. . $00000 \odot \odot ० \odot$

๑. . $0000000 \odot \odot$

๑. 000000000

0.000000000

3. 065437000

$-3.065437000$

0.000000000

$\odot .000000000$

$\odot .000000000$

$\odot .000000 \odot \odot \odot$

$\odot .000000000$

$\odot .000000000$

๑ . $00000000 \odot$

○. 000000000

๑. . 000000000

$\odot .0000000 \odot \odot$
- 0.264546000

$-1.094625000$

3. 061486000

$-2.406932000$

3.957623000

1. 795594000

- 3.036552000

$-0.764727000$

3. 189007000

$\odot .593296000$

$-2.039846000$

3. 061486000

$-2.406932000$

3.957623000

1. 795594000

$-3.036552000$

$-0.764727000$

3.189007000

0.593296000

$-2.039846000$

$-4.061674000$

$-0.466332000$

$-0.466332000$

$-1.739989000$

$-1.739989000$

5.034705000

$-4.098611000$

3.560773000

$\odot .703611000$

$-2.172836000$

$-1.739989000$

$-1.739989000$

5.034705000

$-4.098611000$

3. 560773000

0.703611000

$-2.172836000$

1.815444000

$-1.068216000$

1.815444000

$-1.068216000$

๑. 240701000

33. $\left\{\operatorname{Re}[\mathrm{Cor}](\mathrm{NMe})_{2}\right\}^{-;}$; 348.35796684 eV
C
0.000000000
C
C
๑. 000000000
$\odot .00000000 \odot$
๑ . $00000000 \odot$

0.000000000

1. 809628000

2.942537000

$-1.809628000$
$-2.963727000$

$-0.264443000$

$-1.184081000$

$-0.264443000$ 


\begin{tabular}{|c|c|}
\hline C & 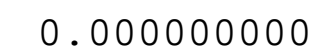 \\
\hline C & ๑. 728055000 \\
\hline C & 1.31204600 \\
\hline C & 1.847792000 \\
\hline C & 2.61743100 \\
\hline C & 2.63130500 \\
\hline $\mathrm{C}$ & 2.88825900 \\
\hline $\mathrm{C}$ & 3.00532200 \\
\hline C & 3.365584000 \\
\hline C & 3.578447000 \\
\hline C & -0.72805500 \\
\hline C & -1.31204600 \\
\hline C & -1.84779200 \\
\hline C & -2.61743100 \\
\hline C & -2.63130500 \\
\hline $\mathrm{C}$ & -2.88825900 \\
\hline $\mathrm{C}$ & -3.00532200 \\
\hline C & -3.365584000 \\
\hline C & -3.57844700 \\
\hline $\mathrm{H}$ & 0.00000000 \\
\hline $\mathrm{H}$ & $\odot .00000000$ \\
\hline $\mathrm{H}$ & $\odot .0000000 \odot \odot$ \\
\hline $\mathrm{H}$ & $\odot .88538500$ \\
\hline $\mathrm{H}$ & 0.88538500 \\
\hline $\mathrm{H}$ & 1.80935000 \\
\hline $\mathrm{H}$ & 2.82160000 \\
\hline $\mathrm{H}$ & 4.018961000 \\
\hline $\mathrm{H}$ & 4.44380000 \\
\hline $\mathrm{H}$ & 4.64980200 \\
\hline $\mathrm{H}$ & -0.88538500 \\
\hline $\mathrm{H}$ & -0.88538500 \\
\hline $\mathrm{H}$ & -1.809350000 \\
\hline $\mathrm{H}$ & -2.82160000 \\
\hline $\mathrm{H}$ & -4.01896100 \\
\hline $\mathrm{H}$ & -4.44380000 \\
\hline $\mathrm{H}$ & -4.64980200 \\
\hline $\mathrm{N}$ & 1.25239300 \\
\hline $\mathrm{N}$ & 1.56805000 \\
\hline $\mathrm{N}$ & -1.25239300 \\
\hline $\mathrm{N}$ & -1.56805000 \\
\hline $\mathrm{Re}$ & $\odot .00 \odot \odot \odot \odot ० \odot$ \\
\hline
\end{tabular}

$-2.942537000$

0.000000000

○. 000000000

๑. 000000000

๑ . $0000000 \odot \odot$

๑ . 000000000

0.000000000

๑. . $0000000 \odot \odot$

๑ . $.00000 \odot ० \odot$

๑. 000000000

$\odot .00000000 \odot$

๑. 000000000

๑. $00000000 \odot$

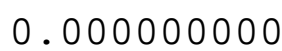

0.000000000

०. $00000000 \odot$

$\odot .00000000 \odot$

$\odot .000000000$

๑. 000000000

๑ . $0000000 \odot \odot$

3. 890257000

- 3.890257000

2. 934037000

$-2.934037000$

๑. 000000000

๑. $00000000 \odot$

๑. $.000 \odot \odot ० ० ० \odot$

0.000000000

๑. $00000 \odot ० ० \odot$

2. 934037000

$-2.934037000$

0.000000000

०. 000000000

๑ . 000000000

๑ . $0000000 \odot \odot$

๑ . $00000000 \odot$

๑. 000000000

$\odot .000000000$

๑ . $000 \odot \odot ० ० ० \odot$

๑. 000000000

$\odot .000000000$
$-1.184081000$

3.101084000

$-2.380563000$

3. 986240000

1.823062000

- 3.006604000

$-0.728372000$

3. 208900000

$\odot .605932000$

- 2.011760000

3. 101084000

$-2.380563000$

3. 986240000

1. 823062000

$-3.006604000$

$-0.728372000$

3. 208900000

$\odot .605932000$

- 2.011760000

$-4.048324000$

$-0.631925000$

$-0.631925000$

$-1.835733000$

$-1.835733000$

5.064855000

$-4.069870000$

3. 581085000

๑. 718816000

$-2.148372000$

$-1.835733000$

$-1.835733000$

5.064855000

$-4.069870000$

3. 581085000

$\odot .718816000$

$-2.148372000$

1.843397000

$-1.045694000$

1.843397000

$-1.045694000$

๑. 270420000

34. $\operatorname{Ir}[\mathrm{Cor}]\left(\mathrm{PMe}_{3}\right) ; C_{s} ; S=0 ;$ Total bonding energy $=-$ $359.51050008 \mathrm{eV}$

$\begin{array}{ll}\text { C } & 0.331003000 \\ \text { C } & 0.331003000 \\ \text { C } & 0.745746000 \\ \text { C } & 0.745746000 \\ \text { C } & 1.543899000\end{array}$

C

C
$-0.485920000$

$-0.485920000$

2.925627000

2.925627000

$-\odot .419116000$
3.319268000

$-3.319268000$

1.440123000

$-1.440123000$

2.606259000 


\begin{tabular}{|c|c|}
\hline C & 1.543899000 \\
\hline C & $2.80268100 \odot$ \\
\hline C & 2.80268100 \\
\hline C & 2.91925400 \\
\hline C & 2.91925400 \\
\hline C & 3.68409000 \\
\hline C & 3.68409000 \\
\hline C & $-\odot .9728600 \odot$ \\
\hline C & $-\odot .97286000$ \\
\hline C & -1.744891000 \\
\hline C & -2.21560700 \\
\hline C & -2.21560700 \\
\hline C & -2.64365600 \\
\hline C & -2.643656000 \\
\hline C & -3.226560000 \\
\hline C & -3.22656000 \\
\hline C & -3.25930600 \\
\hline $\mathrm{H}$ & $\odot .280889000$ \\
\hline $\mathrm{H}$ & ๑. 28088900 \\
\hline $\mathrm{H}$ & 0.40066400 \\
\hline $\mathrm{H}$ & 0.40066400 \\
\hline $\mathrm{H}$ & 0.63956200 \\
\hline $\mathrm{H}$ & ๑. 639562000 \\
\hline $\mathrm{H}$ & 1.80158600 \\
\hline $\mathrm{H}$ & 1.80158600 \\
\hline $\mathrm{H}$ & 3.28158000 \\
\hline $\mathrm{H}$ & 3.281580000 \\
\hline $\mathrm{H}$ & 4.75105200 \\
\hline $\mathrm{H}$ & 4.75105200 \\
\hline $\mathrm{H}$ & -1.71072400 \\
\hline $\mathrm{H}$ & -2.27798300 \\
\hline $\mathrm{H}$ & -2.27798300 \\
\hline $\mathrm{H}$ & -2.30490800 \\
\hline $\mathrm{H}$ & -2.30490800 \\
\hline $\mathrm{H}$ & -4.27310000 \\
\hline $\mathrm{H}$ & -4.27310000 \\
\hline $\mathrm{H}$ & -4.33094700 \\
\hline $\operatorname{Ir}$ & 0.03382000 \\
\hline $\mathrm{N}$ & 1.54701700 \\
\hline $\mathrm{N}$ & 1.54701700 \\
\hline $\mathrm{N}$ & -1.29460000 \\
\hline $\mathrm{N}$ & -1.29460000 \\
\hline$P$ & -0.05079006 \\
\hline
\end{tabular}

$-0.419116000$

$-0.370478000$

$-0.370478000$

$-0.603506000$

$-0.603506000$

$-0.578887000$

$-0.578887000$

$-0.493105000$

- $\odot .493105000$

2. 812360000

$-0.711022000$

$-0.711022000$

$-0.565302000$

$-0.565302000$

$-0.754488000$

$-0.754488000$

$-0.613066000$

2. 562793000

2.562793000

$-0.618064000$

$-0.618064000$

4.009968000

4. 009968000

2. 661263000

2. 661263000

$-0.770190000$

$-0.77019000 \odot$

$-0.723599000$

$-0.723599000$

3.903270000

2. 464292000

2.464292000

$-0.848160000$

$-0.848160000$

$-0.931921000$

$-\odot .931921000$

$-0.767627000$

$-0.051007000$

$-\odot .253409000$

$-0.253409000$

$-0.390066000$

$-0.390066000$

2. 136786000
$-2.606259000$

$\odot .718932000$

$-0.718932000$

2. 978910000

$-2.978910000$

1.823420000

$-1.823420000$

2.788921000

$-2.788921000$

๑. . 000000000

3. 487159000

$-3.487159000$

1. 261942000

$-1.261942000$

2.567064000

$-2.567064000$

0.000000000

2.355772000

$-2.355772000$

4. 391957000

$-4.391957000$

1.379714000

$-1.379714000$

1. 461346000

$-1.461346000$

3.980991000

$-3.980991000$

1. 763616000

$-1.763616000$

0.000000000

$\odot .883253000$

$-0.883253000$

4.553754000

$-4.553754000$

2.758558000

$-2.758558000$

๑ . 000000000

$\odot .000000000$

1. 250018000

$-1.250018000$

1. 447748000

$-1.447748000$

$0.00000000 \odot$

35. $\operatorname{Ir}[$ Cor $](\mathrm{CO}) ; C_{\mathrm{s}} ; S=0$; Total bonding energy = - $303.46545253 \mathrm{eV}$
C
0.134358000
$-0.723388000$
2.784710000
$-0.723388000$
$-2.784710000$
C
$\odot .134358000$
$\odot .515121000$
3. 322387000 


\begin{tabular}{|c|c|}
\hline C & $\odot .522062000$ \\
\hline C & $\odot .94301900 \odot$ \\
\hline C & ๑. 943019000 \\
\hline C & 1.14557300 \\
\hline C & 1.14557300 \\
\hline C & 1.341118000 \\
\hline C & 1.341118000 \\
\hline C & 1.38136500 \\
\hline C & 1.38136500 \\
\hline C & 2.65825000 \\
\hline C & -0.424816000 \\
\hline C & -0.424816000 \\
\hline C & $-\odot .44591400$ \\
\hline C & -0.44591400 \\
\hline C & -0.65300200 \\
\hline C & -0.78407600 \\
\hline C & -0.78407600 \\
\hline $\mathrm{H}$ & $\odot .413100000$ \\
\hline $\mathrm{H}$ & 0.413100000 \\
\hline $\mathrm{H}$ & 1.08270000 \\
\hline $\mathrm{H}$ & 1.08270000 \\
\hline $\mathrm{H}$ & 1.53432700 \\
\hline $\mathrm{H}$ & 1.53432700 \\
\hline $\mathrm{H}$ & $-\odot .60739700$ \\
\hline $\mathrm{H}$ & $-\odot .6073970 \odot$ \\
\hline $\mathrm{H}$ & -1.11503200 \\
\hline $\mathrm{H}$ & -1.26396700 \\
\hline $\mathrm{H}$ & -1.26396700 \\
\hline $\operatorname{Ir}$ & ๑. 97010400 \\
\hline $\mathrm{N}$ & $\odot .14044800$ \\
\hline $\mathrm{N}$ & 0.14044800 \\
\hline $\mathrm{N}$ & 1.10339300 \\
\hline $\mathrm{N}$ & 1.10339300 \\
\hline 0 & 3.73342100 \\
\hline
\end{tabular}

๑. 515121000

1.642728000

1. 642728000

3. 014838000

3. 014838000

2.829202000

2.829202000

3. 736868000

3. 736868000

$-0.570935000$

$-2.294987000$

$-2.294987000$

$-1.841140000$

$-1.841140000$

$-2.868517000$

$-2.792562000$

$-2.792562000$

0.621955000

$\odot .621955000$

3. 411805000

3. 411805000

4.801988000

4.801988000

$-1.884183000$

$-1.884183000$

$-3.847268000$

$-3.740001000$

$-3.740001000$

0.059519000

$-1.051175000$

$-1.051175000$

1.589576000

1.589576000

$-0.983520000$
$-3.322387000$

2. 606008000

$-2.606008000$

2.968847000

$-2.968847000$

$\odot .714048000$

- $\odot .714048000$

1.812850000

$-1.812850000$

0.000000000

1. 256337000

$-1.256337000$

3.476887000

$-3.476887000$

0.000000000

2.554524000

$-2.554524000$

4.394051000

$-4.394051000$

3.969335000

$-3.969335000$

1.746294000

$-1.746294000$

4.542556000

$-4.542556000$

๑. . 000000000

2. 741795000

$-2.741795000$

$\odot .000 \odot \odot \odot \odot \odot \odot$

1.440427000

$-1.440427000$

1.245655000

$-1.245655000$

0.000000000

36. $\operatorname{Ir}[\mathrm{Cor}](\mathrm{CO})\left(\mathrm{PMe}_{3}\right)$ $378.13715171 \mathrm{eV}$

$\begin{array}{lr}\text { C } & 0.657593000 \\ \text { C } & 0.942562000 \\ \text { C } & 1.099307000 \\ \text { C } & 1.099307000 \\ \text { C } & 2.323048000 \\ \text { C } & 2.323048000 \\ \text { C } & 2.736732000 \\ \text { C } & 2.736732000 \\ \text { C } & 3.311215000 \\ \text { C } & 3.311215000 \\ \text { C } & 3.337853000 \\ \text { C } & -0.174776000\end{array}$

2.267895000
$-3.09506800 \odot$
$0.09151900 \odot$
$0.09151900 \odot$
-0.214149000
-0.214149000
$-0.28826400 \odot$
$-0.28826400 \odot$
$-0.44120200 \odot$
$-0.44120200 \odot$
-0.421022000
$0.40139800 \odot$
0.000000000

0.000000000

2.806237000

$-2.806237000$

3. 513183000

$-3.513183000$

1. 273133000

$-1.273133000$

2.592213000

$-2.592213000$

0.000000000

3.328589000 


\begin{tabular}{|c|c|}
\hline C & -0.174776000 \\
\hline C & -1.367512000 \\
\hline C & -1.36751200 \\
\hline C & -1.47426400 \\
\hline C & -1.474264000 \\
\hline C & -2.607966000 \\
\hline C & -2.60796600 \\
\hline C & -2.73125100 \\
\hline C & -2.73125100 \\
\hline C & -3.485776000 \\
\hline C & -3.48577600 \\
\hline $\mathrm{H}$ & 0.59311200 \\
\hline $\mathrm{H}$ & 1.55426000 \\
\hline $\mathrm{H}$ & 1.554260000 \\
\hline $\mathrm{H}$ & 2.422507000 \\
\hline $\mathrm{H}$ & 2.42250700 \\
\hline $\mathrm{H}$ & 4.34107800 \\
\hline $\mathrm{H}$ & 4.341078000 \\
\hline $\mathrm{H}$ & 4.39279100 \\
\hline $\mathrm{H}$ & $-\odot .2465990 \odot$ \\
\hline $\mathrm{H}$ & $-\odot .2465990 \odot$ \\
\hline $\mathrm{H}$ & $-\odot .9211170 \odot$ \\
\hline $\mathrm{H}$ & -0.921117000 \\
\hline $\mathrm{H}$ & -1.69407100 \\
\hline $\mathrm{H}$ & -1.69407100 \\
\hline $\mathrm{H}$ & -2.40640300 \\
\hline $\mathrm{H}$ & -2.40640300 \\
\hline $\mathrm{H}$ & -3.10196900 \\
\hline $\mathrm{H}$ & -3.10196900 \\
\hline $\mathrm{H}$ & -4.54489500 \\
\hline $\mathrm{H}$ & -4.54489500 \\
\hline $\operatorname{Ir}$ & $\odot .12593200$ \\
\hline $\mathrm{N}$ & 1.42429400 \\
\hline $\mathrm{N}$ & 1.42429400 \\
\hline $\mathrm{N}$ & -1.37282300 \\
\hline $\mathrm{N}$ & -1.37282300 \\
\hline 0 & 1.00004400 \\
\hline$P$ & -0.48486700 \\
\hline
\end{tabular}

๑. 401398000

$\odot .697301000$

$\odot .697301000$

$-2.492150000$

$-2.492150000$

$\odot .974852000$

$\odot .974852000$

๑. 943471000

$\odot .943471000$

1. 109128000

1. 109128000

$-4.128529000$

$-2.918462000$

$-2.918462000$

$-\odot .248126000$

$-0.248126000$

$-\odot .688599000$

$-0.688599000$

$-0.662575000$

๑. 402649000

๑. 402649000

$-2.298063000$

- 2.298063000

$-3.558075000$

$-3.558075000$

$-1.930159000$

$-1.930159000$

$\odot .988209000$

๑. 988209000

1. 306292000

1. 306292000

0.441136000

๑. 018778000

๑ . 018778000

$\odot .741548000$

$\odot .741548000$

3. 353168000

$-1.956115000$
$-3.328589000$

2. 614642000

$-2.614642000$

1. 439502000

$-1.439502000$

0.724162000

$-\odot .724162000$

2. 998994000

- 2.998994000

1.843840000

$-1.843840000$

0.000000000

$\odot .882925000$

$-0.882925000$

4.587063000

$-4.587063000$

2. 796429000

$-2.796429000$

๑. . 000000000

4.408979000

$-4.408979000$

2.357074000

$-2.357074000$

1. 364490000

$-1.364490000$

1.476527000

$-1.476527000$

4 . 010866000

$-4.010866000$

1.800572000

$-1.800572000$

0.000000000

1.469826000

$-1.469826000$

1. 258775000

$-1.258775000$

๑ . 000000000

๑. . 000000000

37. $\operatorname{Re}[\mathrm{Cor}](\mathrm{NMe}) ; C_{\mathrm{s}} ; s=0 ;$ Total bonding energy = $320.21619559 \mathrm{eV}$

$\begin{array}{ll}\text { C } & 0.433103000 \\ \text { C } & 0.433103000 \\ \text { C } & 1.612914000 \\ \text { C } & 1.612914000 \\ \text { C } & 2.843907000 \\ \text { C } & 2.843907000 \\ \text { C } & 2.977511000 \\ \text { C } & 2.977511000\end{array}$

$-0.014594000$

$-0.014594000$

๑. 087585000

๑ . 087585000

$\odot .133599000$

$\odot .133599000$

$-0.118909000$

$-\odot .11890900 \odot$
3.328243000

$-3.328243000$

2. 589382000

$-2.589382000$

0.706160000

$-0.706160000$

2. 944910000

$-2.944910000$ 


\begin{tabular}{|c|c|}
\hline C & 3.731695000 \\
\hline C & 3.731695000 \\
\hline $\mathrm{C}$ & -0.27011000 \\
\hline C & -0.84457000 \\
\hline C & $-\odot .84457000 \odot$ \\
\hline $\mathrm{C}$ & -2.066796000 \\
\hline $\mathrm{C}$ & -2.06679600 \\
\hline $\mathrm{C}$ & -2.48671500 \\
\hline C & -2.486715000 \\
\hline C & -3.065612000 \\
\hline C & -3.06561200 \\
\hline $\mathrm{C}$ & -3.11207800 \\
\hline $\mathrm{H}$ & ๑. 510181000 \\
\hline $\mathrm{H}$ & ๑. 51018100 \\
\hline $\mathrm{H}$ & ๑. 69834100 \\
\hline $\mathrm{H}$ & 3.34139800 \\
\hline $\mathrm{H}$ & 3.34139800 \\
\hline $\mathrm{H}$ & 4.79390900 \\
\hline $\mathrm{H}$ & 4.79390900 \\
\hline $\mathrm{H}$ & -0.82605300 \\
\hline $\mathrm{H}$ & $-\odot .8260530 \odot$ \\
\hline $\mathrm{H}$ & -2.15032300 \\
\hline $\mathrm{H}$ & -2.15032300 \\
\hline $\mathrm{H}$ & -4.10444500 \\
\hline $\mathrm{H}$ & -4.10444500 \\
\hline $\mathrm{H}$ & -4.17154100 \\
\hline $\mathrm{N}$ & 1.57542700 \\
\hline $\mathrm{N}$ & 1.57542700 \\
\hline $\mathrm{N}$ & -0.08767300 \\
\hline $\mathrm{N}$ & -1.13622500 \\
\hline $\mathrm{N}$ & -1.13622500 \\
\hline $\mathrm{Re}$ & 0.088947 \\
\hline
\end{tabular}

$-0.101256000$

$-0.101256000$

3. 995997000

- 0.052905000

- 0.052905000

$-0.341126000$

$-0.341126000$

$-0.194006000$

- $\odot .194006000$

$-0.430483000$

$-0.430483000$

$-0.279016000$

$-0.163782000$

$-0.163782000$

4.502489000

$-0.299142000$

$-0.299142000$

$-0.268141000$

- $\odot .268141000$

4. 306911000

4.306911000

$-\odot .495578000$

$-0.495578000$

$-0.662035000$

$-0.662035000$

- 0.498851000

๑. 285059000

๑. 285059000

2. 582869000

0.069316000

๑. 069316000

$\odot .882000000$
1.782216000

$-1.782216000$

0.000000000

2.772477000

$-2.772477000$

3. 455866000

- 3.455866000

1. 241318000

$-1.241318000$

2. 526067000

$-2.526067000$

0.000000000

4.397154000

$-4.397154000$

0.000000000

3. 944129000

$-3.944129000$

1.703678000

$-1.703678000$

๑. 888020000

$-0.888020000$

4.520387000

$-4.520387000$

2. 700797000

$-2.700797000$

0.000000000

1. 229166000

$-1.229166000$

๑. . $0 \odot \odot \odot ० ० ० \odot \odot$

1.413807000

$-1.413807000$

$0.00000000 \odot$

38. $\mathrm{Me}_{3} \mathrm{PO} ; C_{s} ; S=0 ;$ Total bonding energy $=-83.70709718 \mathrm{eV}$

$\begin{array}{lr}\mathrm{C} & 0.995842000 \\ \mathrm{C} & -1.182218000 \\ \mathrm{C} & -1.182218000 \\ \mathrm{H} & 0.432962000 \\ \mathrm{H} & 1.632953000 \\ \mathrm{H} & 1.632953000 \\ \mathrm{H} & -0.579461000 \\ \mathrm{H} & -0.579461000 \\ \mathrm{H} & -1.704515000 \\ \mathrm{H} & -1.704515000 \\ \mathrm{H} & -1.911826000 \\ \mathrm{H} & -1.911826000 \\ \mathrm{O} & 0.635838000 \\ \mathrm{P} & -0.096500000\end{array}$

1.264531000

0.029278000

0.029278000

2.198673000

1.226175000

1.226175000

$-0.031299000$

$-\odot .031299000$

0.986435000

0.986435000

$-0.780710000$

$-0.780710000$

$-1.485512000$

- $.19334700 \odot$
0.000000000

1.444386000

$-1.444386000$

0.000000000

$\odot .883779000$

$-0.883779000$

2. 350846000

$-2.350846000$

1.421408000

$-1.421408000$

1.463699000

$-1.463699000$

0.0000000000

0.0000000000 
39. $C_{0} ; C_{\text {lin }} ; S=0 ;$ Total bonding energy $=-18.10684518 \mathrm{eV}$
C
0.000000000
$\odot .000000000$
$-1.637338000$
0
0.0000000000
0.000000000
$-2.762881000$

40. $\mathrm{PMe}_{3} ; C_{\mathrm{s}} ; S=0$; Total bonding energy $=-73.88983796 \mathrm{eV}$

$\begin{array}{rr}\mathrm{C} & 0.972749000 \\ \mathrm{C} & 0.972749000 \\ \mathrm{C} & -1.471666000 \\ \mathrm{H} & 0.471479000 \\ \mathrm{H} & 0.471479000 \\ \mathrm{H} & 1.031035000 \\ \mathrm{H} & 1.031035000 \\ \mathrm{H} & 1.984721000 \\ \mathrm{H} & 1.984721000 \\ \mathrm{H} & -1.252313000 \\ \mathrm{H} & -2.069315000 \\ \mathrm{H} & -2.069315000 \\ \mathrm{P} & 0.063786000\end{array}$

0.148507000

0.148507000

0.406311000

$-0.100480000$

$-0.100480000$

1.236039000

1.236039000

$-0.256067000$

$-0.256067000$

1.476966000

0.167932000

0.167932000

$-0.634194000$
1.415358000

$-1.415358000$

0.000000000

2. 351693000

$-2.351693000$

1.322991000

$-1.322991000$

1.462973000

$-1.462973000$

0.000000000

0.880980000

$-0.880980000$

0.000000000
41. $\mathrm{MeN}=\mathrm{NMe} ; C_{2 \mathrm{~h}} ; S=0$

\section{C}

C

$\mathrm{H}$

$\mathrm{H}$

$\mathrm{H}$

$\mathrm{H}$

$\mathrm{H}$

$\mathrm{H}$

$\mathrm{N}$

$\mathrm{N}$
1.798822000

$-1.798822000$

1.833834000

2. 305681000

2. 305681000

$-1.833834000$

$-2.305681000$

$-2.305681000$

0.426973000

$-\odot .426973000$
Total bonding

- 0.063912000

0.063912000

$-1.155606000$

0.339184000

0.339184000

1.155606000

$-0.339184000$

$-\odot .339184000$

0.444461000

$-0.444461000$ energy $=-63.42932681 \mathrm{eV}$

0.000000000

0.000000000

0.000000000

0.879000000

$-\odot .879000000$

0.000000000

0.879000000

$-0.879000000$

0.000000000

0.000000000

42. $\mathrm{Me}_{3} \mathrm{P}=\mathrm{NMe} ; C_{1} ; S=0 ;$ Total bonding energy $=-105.95446898$ $\mathrm{eV}$

\begin{tabular}{|c|c|}
\hline C & $1.0073080 \odot \odot$ \\
\hline C & 1.901978000 \\
\hline c & -0.937133000 \\
\hline C & -1.139551000 \\
\hline H & $\odot .35851100 \odot$ \\
\hline 1 & 1.556419000 \\
\hline $\mathrm{H}$ & 1.578395000 \\
\hline $\mathrm{H}$ & $1.727740000 \odot$ \\
\hline $\mathrm{H}$ & $2.25644600 \odot$ \\
\hline $\mathrm{H}$ & 2.780714000 \\
\hline $\mathrm{H}$ & $-\odot .27462000 \odot$ \\
\hline 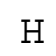 & $-\odot .60122400 \odot$ \\
\hline & -1.48854800 \\
\hline
\end{tabular}

1.343661000

$-2.095207000$

$-0.155193000$

0.007391000

2.206933000

1.467048000

$-2.353892000$

1.295450000

$-3.022003000$

$-1.440340000$

$-0.253683000$

0.054544000

$\odot .782097000$ $-\odot .056189000$

$\odot .411974000$

1.417840000

$-1.466213000$

0.099113000

- $\odot .989841000$

1.431104000

0.761544000

$-0.045125000$

$\odot .510719000$

2. 278867000

$-2.411825000$

1. 505350000 


$\begin{array}{rrrr}\mathrm{H} & -1.63841600 \odot & -0.98972100 \odot & 1.42702800 \odot \\ \mathrm{H} & -1.7156400 \odot \odot & 0.92233800 \odot & -1.32560400 \odot \\ \mathrm{H} & -1.81217900 \odot & -0.84875700 \odot & -1.49375200 \odot \\ \mathrm{N} & 0.84910900 \odot & -1.53551000 \odot & -0.40951900 \odot \\ \mathrm{P} & 0.05715900 \odot & -0.230065000 & -0.12719500 \odot\end{array}$

